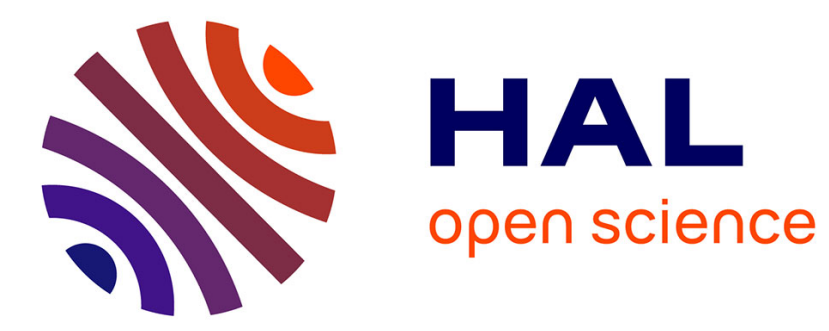

\title{
Etude de la flore de levure dans le fromage de Roquefort
}

\author{
M. Galzin, P. Galzy, G. Bret, S. Bentchicou, J. P. Lauzet
}

\section{To cite this version:}

M. Galzin, P. Galzy, G. Bret, S. Bentchicou, J. P. Lauzet. Etude de la flore de levure dans le fromage de Roquefort. Le Lait, 1970, 50 (491_492), pp.1-37. hal-00928504

\section{HAL Id: hal-00928504 https://hal.science/hal-00928504}

Submitted on 1 Jan 1970

HAL is a multi-disciplinary open access archive for the deposit and dissemination of scientific research documents, whether they are published or not. The documents may come from teaching and research institutions in France or abroad, or from public or private research centers.
L'archive ouverte pluridisciplinaire HAL, est destinée au dépôt et à la diffusion de documents scientifiques de niveau recherche, publiés ou non, émanant des établissements d'enseignement et de recherche français ou étrangers, des laboratoires publics ou privés. 


\title{
Etude de la flore de levure dans le fromage de Roquefort
}

\author{
par \\ M. GALZIN, P. GALZY (*) et G. BRET \\ (avec la collaboration technique de S. BENTCHICOU et J. P. LAUZET) \\ Laboratoires de la Société des Caves (12) Roquefort \\ et \\ (*) Laboratoire de Recherches de la Chaire de Génétique \\ CRAM. INRA. ENSA (34) Montpellier
}

Les levures sont très répandues dans les fromages fermiers. Leur rôle est peu connu car les travaux ont porté surtout sur les bactéries lactiques. Cependant, V. Maxa et V. Jicinscky (1956), ont montré que dans les fromages Bleu et le Roquefort, les levures jouaient un rôle important dans la formation du goût et de l'arôme. Il faut citer aussi les travaux de J. Proks et coll. (1959) et les travaux de J. Dolezalek et M. Bartuskova (1966).

En outre, les levures à métabolisme fermentatif contribuent à la formation de l'ouverture dans le Roquefort et les fromages Bleu.

Enfin, des essais de fabrication de Roquefort, avec et sans Penicillium roqueforti, ont montré que les levures jouent un rôle dans la lipolyse et la protéolyse, et que les levures et le $P$. roqueforti ont des actions complémentaires dans la formation des produits aromatiques.

Le rôle des bactéries, dans la fabrication et l'affinage du Roquefort a été précisé par les travaux de Devoyod et coll. (1966-1968-1969) mais le rôle des levures restait peu connu. suivant.

Ces considérations nous ont conduit à entreprendre le travail

La flore de levure a été étudiée dans un cuvier depuis la fabrication jusqu'à la fin de l'affinage.

\section{I. - MATERIEL ET METHODES}

\section{a) Stades de prélèvement}

Nous avons fait des prélèvements pour effectuer des analyses microbiologiques, en particulier des analyses de levures, et des analyses chimiques, aux stades importants de l'évolution du fromage. 
- Fabrication :

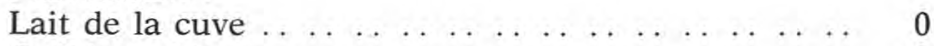

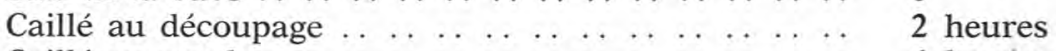

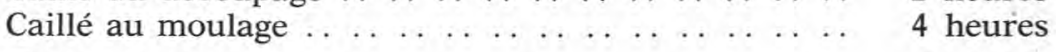

- Egouttage et salage :

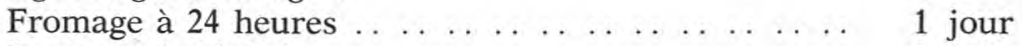

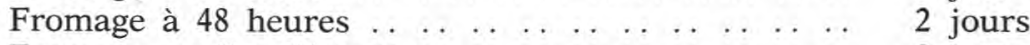

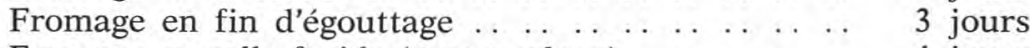

Fromage en salle froide (avant salage) . . . . . . . 4 jours

- Premier affinage:

Fromage, 3 jours après la fin du salage . . . . . . . 11 jours

Fromage, 10 jours après la fin du salage ... . . . . 23 jours

Fromage, 17 jours après la fin du salage . . . . . . . 28 jours

Fromage au plombage (pliage sous étain) . . . . . . 36 jours

- Deuxième affinage :

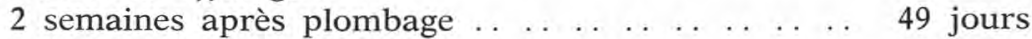

1 mois après plombage . . . . . . . . . . . . . . . . 65 jours

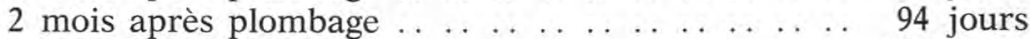

3 mois après plombage . . . . . . . . . . . . . . . . . . . 142 jours

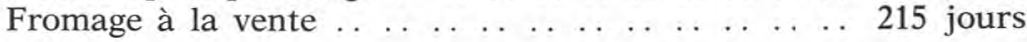

Il est important de noter que les prélèvements étaient faits à chaque stade, sur un fromage différent. Or, des essais précis nous ont montré que les fromages fabriqués à partir du même lait, dans la même cuve, étaient hétérogènes entre eux. Cette hétérogénéité se manifeste surtout aux premiers stades de la fabrication et concerne principalement l'évolution microbiologique, et, par voie de conséquence physico-chimique.

Nous tiendrons compte de ce fait dans l'étude de l'évolution de la flore de levure et de l'évolution générale du cuvier.

\section{b) Technique d'isolement et de culture}

Nous avons utilisé 8 milieux d'isolement. Le milieu de base est constitué par :

- 0,5 p. 100 d'extrait de levure.

- 3 ou 4 p. 100 de gélose, selon le pH du milieu.

Les huit milieux se différencient par:

_ 2 sources de carbone . . l'acide lactique à 2 p. 100 .. L le glucose à 2 p. $100 \quad \ldots \quad \ldots \quad$ G

- $2 \mathrm{pH}$. . 3,5 avec un tampon bitartrate de Potassium L 3 et G 3 5,5 avec un tampon Phosphate . . . . . . . L L 5 et $\mathrm{G} 5$

- 2 taux de sel .. 0 p. $100 \ldots$... L 30 et L 50 - G 30 et G 50 (chlorure de sodium) 10 p. 100 ... . L 310 et L 510 - G 310 et G 510

Soit 8 milieux de composition différente. 
Pour chaque milieu, nous avons ajouté $3 \mu \mathrm{g}$ de 2-(4' Thiazolyl) Benzimidazole ou Thiabendazole par $\mathrm{ml}$, pour eviter l'envahissement du milieu par le $P$. roqueforti.

En effet, cette molécule a des propriétés antifongiques remarquables qui ont été mises en évidence par le Laboratoire de la Société des Caves de Roquefort (Bret et coll., 1963-1964), au cours d'essais réalisés en collaboration avec la Société Chimique Merck Sharp et Dohme, France. double.

- Sur lait et sur caillé, les prélèvements ont été faits en

- Sur fromage, les prélèvements sur les faces se faisaient par grattage, après élimination du révérun (morge du fromage). deux.

$\mathrm{Au}$ centre, nous avons prélevé après avoir coupé le fromage en

Les dilutions convenables étaient étalées sur les 8 milieux de culture, à raison de $0,1 \mathrm{ml}$ de dilution par boîte de Pétri. Après une incubation à $25^{\circ} \mathrm{C}$ de $48 \mathrm{~h}$ pour les milieux non salés et de $72 \mathrm{~h}$ pour les milieux salés, une numération était faite sur chaque boîte. Nous avons prélevé ensuite de 8 à 12 colonies par boîte. Ces colonies étaient choisies proportionnellement aux types coloniaux présents sur la boîte.

Après la numération, nous notons la dilution, la répartition des levures entre les différents types coloniaux, et le nombre de levures de chaque type prélevé. Au dernier stade des prélèvements, c'est-àdire à la vente, nous avions prélevé un peu plus de 3000 souches de levures. L'entretien et le repiquage étaient faits sur le milieu suivant :

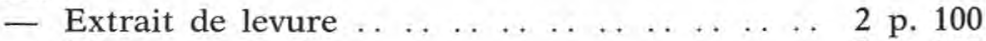

- Glucose .. . . . .

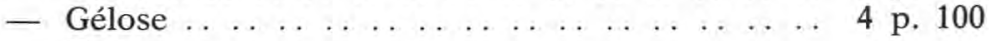

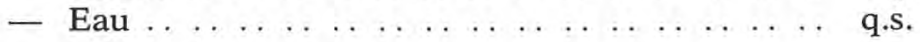

\section{c) Tests taxonomiques}

Chaque souche de levure a été testée en utilisant les techniques de Lodder et Kreger van Rij (1952).

La formation de spores est recherchée sur le milieu Fowel (1967). Il est composé de :

- Acétate de sodium anhydre . . . . . . . . . .

- Chlorure de Potassium ............. 1 p. 100

- Gélose .......................... . . 1,5 p. 100

- Eau distillée . . . . . . . . . . . . . . . . . . . . . $100 \mathrm{ml}$

Le $\mathrm{pH}$ est ajusté à 9 avec de la potasse. Il est ensemencé en surface à partir d'une anse de culture émulsionnée dans $9 \mathrm{ml}$ de sérum physiologique.

L'assimilation du nitrate est testée sur "Yeast Carbon Base " (Difco) additionné de 0,078 p. 100 de Nitrate de Potassium. 


\section{d) Méthodes analytiques}

Les mesures et analyses suivantes sont effectuées sur les prélèvements servant aux numérations des levures et des bactéries :

- Mesure électrométrique directe du $\mathrm{pH}$ avec une électrode de verre.

- Détermination de l'extrait sec par la méthode au sable à $102-105^{\circ} \mathrm{C}$.

- Dosage des chlorures par la méthode de Wilster et al. (1937).

- Dosage du lactose et des sucres réducteurs par la méthode réductimétrique au ferricyanure définie par Hoffman (1937).

Les standards nécessaires à ce dosage sont analysés par la méthode de Somogyi (1952).

- Dosage de l'acide lactique total par la méthode colorimétrique de Ling (1951) adaptée au fromage par Harper et Randolph (1960).

Les deux analyses précédentes sont faites sur appareil Technicon.

- Mesure de la composition gazeuse par chromatographie sur FM 810, équipé de colonnes à tamis moléculaire et silicagel.

\section{e) Techniques bactériologiques}

Un $\mathrm{g}$ de fromage additionné d'environ $0,1 \mathrm{~g}$ de citrate trisodique stérile est broyé dans un mortier stérile et dilué avec $9 \mathrm{ml}$ de solution stérile de Ringer au 1/4. Les dilutions décimales sont faites ensuite avec la solution de Ringer.

- Les germes totaux sont dénombrés sur milieu Plate Count Agar (Difco $n^{\circ} 479$ ) additionné de pourpre de bromocrésol à 0,02 p. 100 (concentration finale) après incubation de $48 \mathrm{~h}$ à $25^{\circ} \mathrm{C}$.

- Les bactéries coliformes sont dénombrées sur Désoxycholate Agar (Difco $\mathrm{n}^{\circ} 420$ ) après incubation $24 \mathrm{~h}$ à $35^{\circ} \mathrm{C}$.

- Les levures sur Potato Dextrose Agar (Difco $n^{\circ} 13$ ) ajusté à $\mathrm{pH} 4$ avec de l'acide tartrique, après incubation de $72 \mathrm{~h}$ à $25^{\circ} \mathrm{C}$.

- Les staphylocoques et les microcoques sur milieu de Chapman (Mannitol salt Agar - Difco no 30 ) après incubation 72 h à $30^{\circ} \mathrm{C}$.

\section{II. - DESCRIPTION DE L'EVOLUTION DU CUVIER}

\section{a) Evolution de la flore}

L'évolution de la flore est indiquée sur les graphiques $\mathrm{n}^{\circ} 1$ pour les faces, et $\mathrm{n}^{\circ} 2$ pour le centre des fromages.

La population totale augmente très rapidement et atteint une valeur maximum au bout de $24 \mathrm{~h}$. Au cours de cette première phase, 
la quantité de tous les types de germes augmente plus ou moins rapidement. La flore dominante est constituée par des bactéries acidifiantes. L'ensemble des autres germes ne représente qu'une partie faible ( 1 à 3 p. 100) de la flore. Au cours du caillage, la population passe en $2 \mathrm{~h}$, de 12 millions à 75 millions, ce qui représente environ 2,5 générations cellulaires. Le grain est ensuite brassé dans le sérum. En $1 \mathrm{~h}$ la population passe alors de 75 millions à 640 millions. La flore reste dans le sérum à une valeur voisine de celle du lait au départ (14 millions). Les bactéries sont surtout présentes dans le grain. C'est ce qui explique que, pendant les brassages, il y a, à partir du grain, une production importante d'acide lactique qui passe dans le sérum. Le lactose du sérum est, par contre, transformé beaucoup plus lentement. L'acidification s'accélère fortement à partir du moulage au moment où le grain est séparé du sérum. La population atteinte en 24 h (13 milliards de germes) est plus forte que celle trouvée par Devoyod et Bret (1964) au Bouyssou (1 milliard). Les valeurs trouvées dans différentes laiteries varient de 1 à 5 milliards de germes. Les valeurs trouvées ici sont donc un peu fortes, mais ne constituent pas une exception parmi les résultats obtenus à la laiterie de Roquefort où s'est effectué cet essai.

Sur les faces du fromage, la population totale diminue en salle chaude, en salle froide et au salage. Elle augmente légèrement ensuite, pour diminuer à nouveau après le plombage. Le nombre de coliformes diminue constamment et devient négligeable après salage. La population de microcoques et staphylocoques diminue en salle chaude et en salle froide, pour augmenter ensuite au cours de l'affinage, de façon continue. Le nombre de levures augmente régulièrement jusqu'au salage, diminue pendant le salage et augmente de nouveau pendant le premier affinage. Des germes alcalinisants se trouvent en général en surface, leur nombre varie de 100 millions à 6 milliards selon le stade d'affinage et selon le fromage. Ils peuvent jouer éventuellement un rôle protéolytique dans les accidents de surface.

Au centre des fromages, la population totale diminue aussi en salle chaude et en salle froide. Le salage a un effet moins marqué et surtout plus lent qu'en surface. Il en résulte une diminution lente mais constante de la flore totale. La flore de coliformes disparaît progressivement mais moins vite qu'en surface. La flore de microcoques et de staphylocoques augmente pendant $24 \mathrm{~h}$, diminue après le salage, augmente légèrement au plombage, mais ces variations sont toujours de faible amplitude. Le nombre de levures augmente jusqu'au salage, se stabilise ensuite et diminue nettement après le plombage.

La flore du révérum présente les caractères généraux de la flore des faces. Elle est simplement beaucoup plus abondante puisqu'elle peut atteindre 80 milliards de germes (tableau $\mathrm{n}^{\circ} 1$ ). 


\section{TABLEAU $N^{\circ} 1$}

Comparaison de la flore du révérun et des faces du fromage

(les populations sont exprimées par le logarithme du nombre de cellules par $\mathrm{g}$ de fromage)

\begin{tabular}{|c|c|c|c|c|c|c|c|}
\hline & & $\begin{array}{l}\text { Flore } \\
\text { totale }\end{array}$ & $\begin{array}{l}\text { Acidi- } \\
\text { fiants }\end{array}$ & $\begin{array}{c}\text { Alcali- } \\
\text { nisants }\end{array}$ & $\begin{array}{l}\text { Micro- } \\
\text { coques }\end{array}$ & $\begin{array}{c}\text { Caséoly- } \\
\text { tiques }\end{array}$ & Levures \\
\hline $\begin{array}{l}\text { Plombage } \\
35^{e} \text { jour }\end{array}$ & $\begin{array}{l}\text { Face } \\
\text { Révérun }\end{array}$ & $\begin{array}{r}9,91 \\
10,90\end{array}$ & $\begin{array}{r}9,26 \\
10,04\end{array}$ & $\begin{array}{l}8,70 \\
9,78\end{array}$ & $\begin{array}{l}6,00 \\
5,00\end{array}$ & $\begin{array}{l}7,00 \\
8,00\end{array}$ & $\begin{array}{r}9,26 \\
10,23\end{array}$ \\
\hline $\begin{array}{l}49^{\circ} \\
\text { jour }\end{array}$ & $\begin{array}{l}\text { Face } \\
\text { Révérun }\end{array}$ & $\begin{array}{r}9,18 \\
10,04\end{array}$ & $\begin{array}{l}8,65 \\
-\end{array}$ & $\begin{array}{l}7,90 \\
9,18\end{array}$ & $\begin{array}{l}8,34 \\
9,28\end{array}$ & $\begin{array}{l}7,00 \\
8,00\end{array}$ & $\begin{array}{r}9,00 \\
10,00\end{array}$ \\
\hline $\begin{array}{c}\text { Vente } \\
197^{\mathrm{e}} \text { jour }\end{array}$ & $\begin{array}{c}\text { Face } \\
\text { Révérun }\end{array}$ & $\begin{array}{l}8,79 \\
9,38\end{array}$ & $\begin{array}{l}8,65 \\
9,26\end{array}$ & $\begin{array}{l}8,18 \\
8,78\end{array}$ & $\begin{array}{l}8,72 \\
9,20\end{array}$ & - & $\begin{array}{l}8,28 \\
8,72\end{array}$ \\
\hline
\end{tabular}

\section{b) Evolution du lactose et de l'acide lactique}

Le graphique $n^{\circ} 3 \mathrm{~A}$, indique les teneurs en lactose du centre et des faces du fromage aux divers stades de la fabrication. Le lactose a pratiquement disparu avant le salage.

Le graphique $\mathrm{n}^{\circ} 3 \mathrm{~A}$, donne aussi les variations de teneur en acide lactique du fromage. La disparition du lactose s'accompagne d'une formation importante d'acide lactique. Le maximum est obtenu en salle froide. Après le salage, la teneur en acide lactique baisse rapidement en surface ; il est probable que les levures sont responsables de la disparition de l'acide lactique. Au centre, la baisse de la teneur en acide lactique est plus lente et commence beaucoup plus tard. Ce fait est à rapprocher d'une observation précédente (graphiques 1 et 2): les bactéries acidifiantes constituent, au centre du fromage, la flore dominante jusqu'à la fin de l'affinage (près de 100 p. 100) alors qu'elle ne constituent guère que le tiers de la flore observée sur les faces et dans le révérum après le salage. Devoyod et Bret (1966) avaient observé une chute d'acidité plus importante au centre des fromages. Il est possible que le centre des fromages ait manqué d'oxygène dans nos essais ce qui pourrait provoquer un retard dans le développement du $P$. roqueforti et des levures. Il est possible aussi que le salage ait eu une action insuffisante car il semble que le sel ait pénétré très lentement dans les fromages étudiés ici.

\section{c) Evolution du $\mathrm{pH}$}

L'évolution du $\mathrm{pH}$ est représentée sur le graphique $\mathrm{n}^{\circ} 3 \mathrm{~B}$. Elle est conforme aux observations effectuées précédemment au cours de la fabrication du Roquefort. Le $\mathrm{pH}$ diminue brutalement pendant 


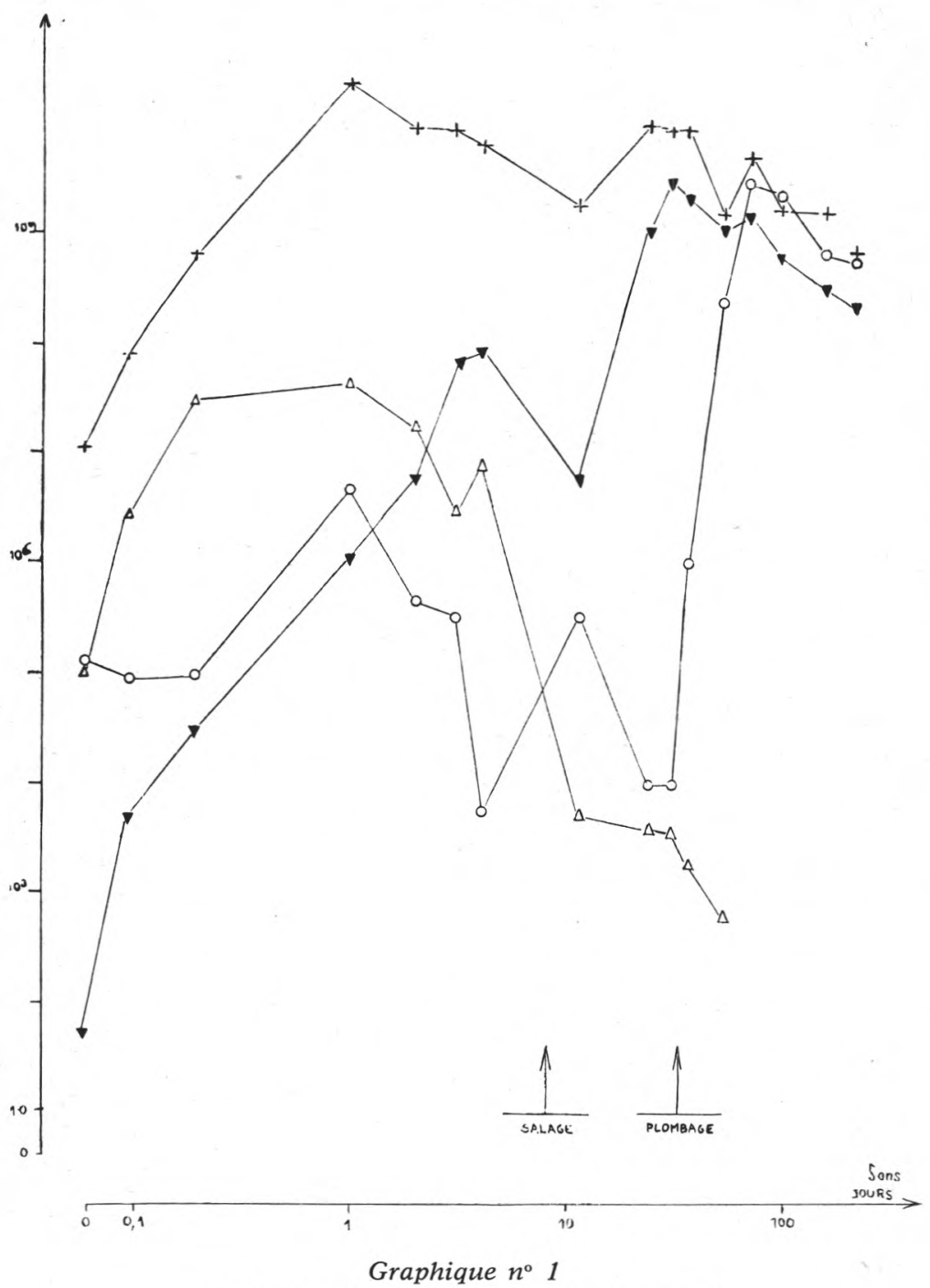

Evolution de la flore (faces du fromage)

Coordonnées logarithmiques.

Abscisses : temps en jours.

Ordonnées : population exprimée en nombre de cellules par g de fromage.

+ population totale

$\triangle$ coliformes

$\nabla$ levures

microcoques staphylocoques 

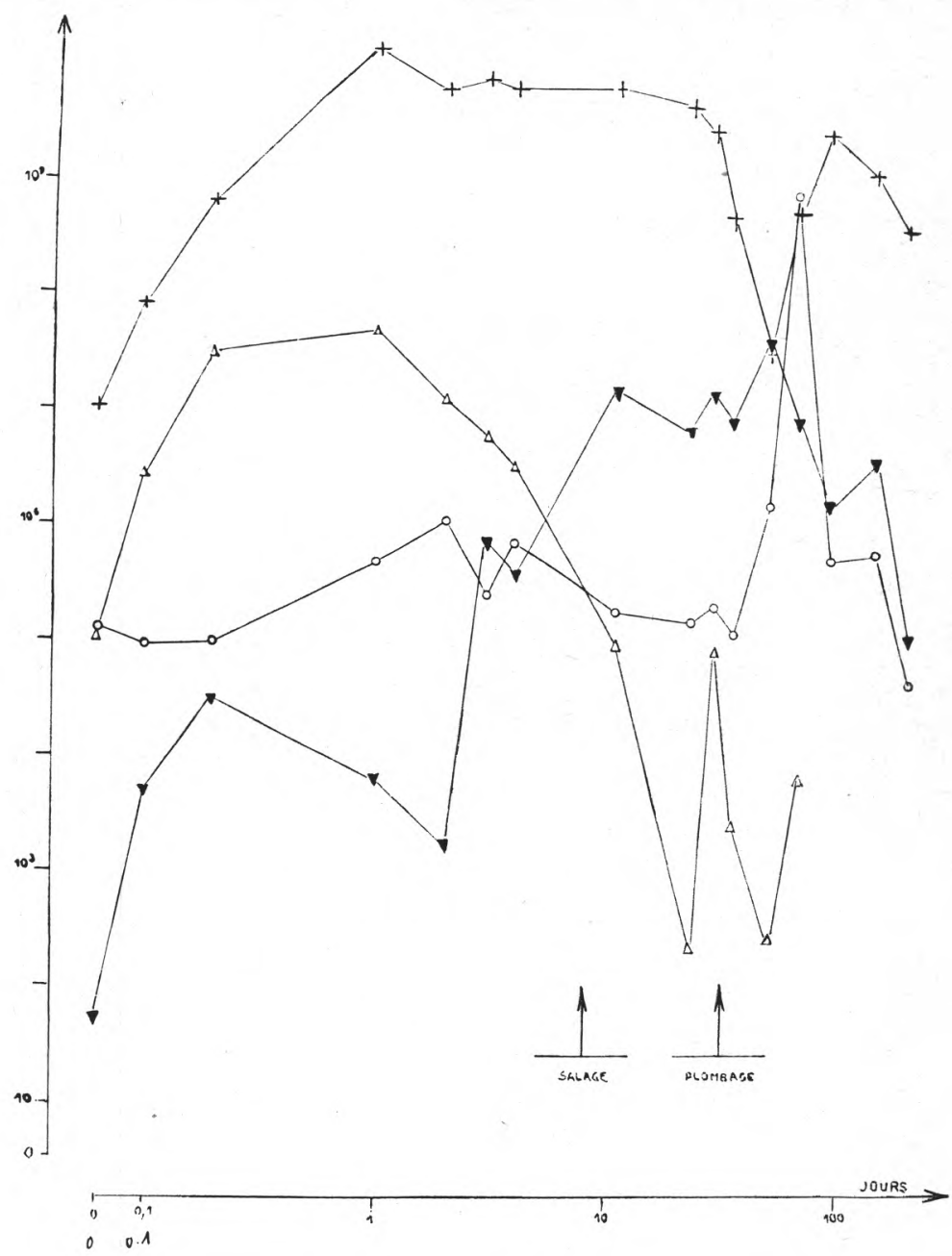

Graphique $n^{\circ} 2$

Evolution de la flore (centre du fromage)

Coordonnées logarithmiques.

Abscisses : temps en jours.

Ordonnées : population exprimée en nombre de cellules par $\mathrm{g}$ de fromage.

+ population totale

$\triangle$ coliformes

$\nabla$ levures

microcoques staphylocoques 


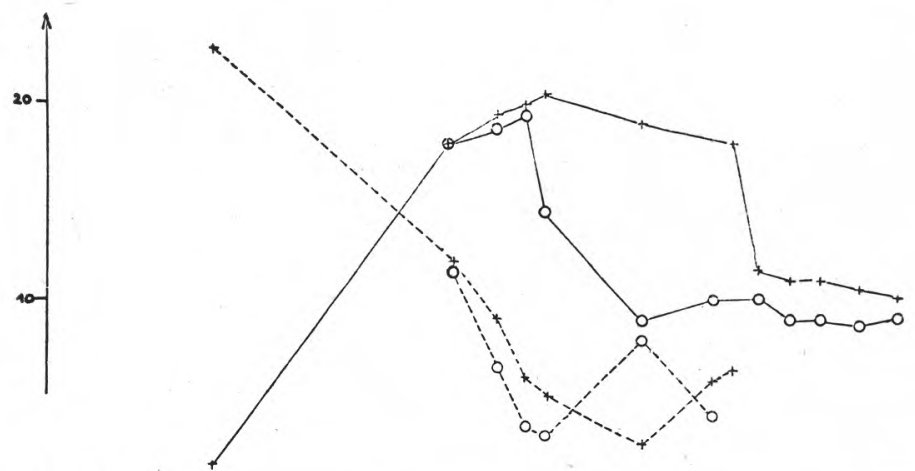

A
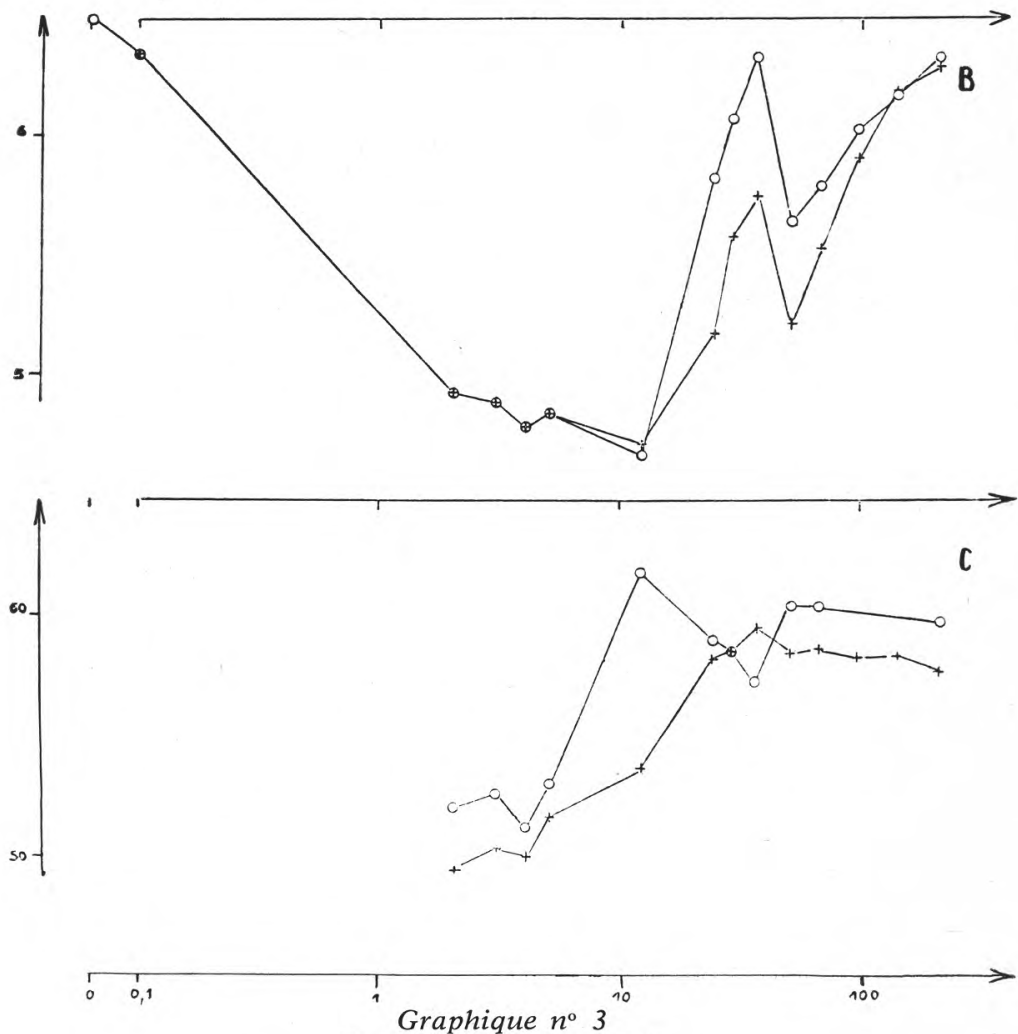

Evolution des fromages

$\mathrm{A}=$ concentration en lactose et acide lactique.

$\mathrm{B}=\mathrm{pH}$

$\mathrm{C}=$ extrait sec.

Abscisses : temps exprimé en jours. Echelle logarithmique.

Ordonnées :

$\mathbf{A}=$ concentrations en lactose et acide lactique, exprimées en $\mathbf{g}$ pour $100 \mathrm{~g}$ de fromage. Echelle arithmétique.

.... lactose

- acide lactique

$+\quad$ centre

faces.

$\mathrm{B}=\mathrm{pH} \quad$ Echelle arithmétique.

$\mathrm{C}=$ extrait sec exprimé en $\mathrm{g}$ pour $100 \mathrm{~g}$ de fromage. Echelle arithmétique. 
$24 \mathrm{~h}$. Après le salage, le $\mathrm{pH}$ s'élève, plus rapidement sur les faces qu'au centre. Ce résultat correspond à une disparition plus rapide de l'acide lactique sur les faces (graphique $n^{\circ} 3 \mathrm{~A}$ ) et s'explique probablement aussi par une activité supérieure des levures en surface.

\section{d) Evolution de l'extrait sec}

Le pourcentage d'extrait sec augmente jusqu'au plombage sur les faces et au centre. Pendant le premier affinage, l'extrait sec des faces devient, provisoirement, inférieur à celui du centre. Ce phénomène a déjà été observé et correspond probablement à une fixation d'eau sur les faces du fromage (graphique $n^{\circ} 3 \mathrm{C}$ ).

\section{e) Evolution des teneurs en oxygène et en gaz carbonique}

Les graphiques $\mathrm{n}^{\circ} 4 \mathrm{~A}$ et $4 \mathrm{~B}$ indiquent, respectivement pour la face et le centre du fromage, la composition de l'atmosphère en pourcentage d'azote, d'oxygène et de gaz carbonique. Les faces contiennent toujours un peu plus d'oxygène et un peu moins de gaz carbonique que le centre. L'évolution est, par contre, parallèle au centre et sur les faces. Dès le $2^{\text {me }}$ jour, la fermentation lactique hétérofermentaire provoque une accumulation de gaz carbonique dans le fromage ; la teneur atteint 75 à 80 p. 100. Pratiquement, la teneur en oxygène est très faible (voisine de 3 p. 100), jusqu'au salage. Le piquage provoque une purge du gaz carbonique et une arrivée brutale d'oxygène. Il en résulte la germination et le développement du $P$. roqueforti d'une part, et le début d'un métabolisme aérobie chez la levure d'autre part. Le métabolisme aérobie de la levure et du $P$. roqueforti est responsable de la disparition de l'acide lactique et de la montée du $\mathrm{pH}$. La teneur en gaz carbonique remonte progressivement du piquage jusqu'au plombage et passe dans le centre du fromage de 7 à 25 p. 100 . Les levures et le $P$. roqueforti sont responsables de ce dégagement de gaz carbonique. A partir du plombage, il s'établit un équilibre entre la diffusion du gaz carbonique du centre vers l'extérieur et l'absorption d'oxygène sur les faces. Cet équilibre se situe entre 20 et 25 p. 100 de gaz carbonique et entre 2 et 4 p. 100 d'oxygène au centre. Pour les faces, les teneurs sont légèrement plus faibles en gaz carbonique.

\section{f) Remarques sur les caractéristiques du cuvier}

Il est permis de considérer que ce cuvier est représentatif de l'évolution normale d'un Roquefort. De l'examen de ces résultats se dégage le rôle de plusieurs facteurs dont la valeur évolue au cours de la maturation du fromage. Ces facteurs : le $\mathrm{pH}$, l'activité de l'eau, le gaz carbonique... conditionnent eux-mêmes cette évolution. Diverses opérations technologiques (acidification, égouttage, salage, piquage, plombage) permettent de diriger et d'orienter l'évolution du fromage par une action importante et parfois brutale. 

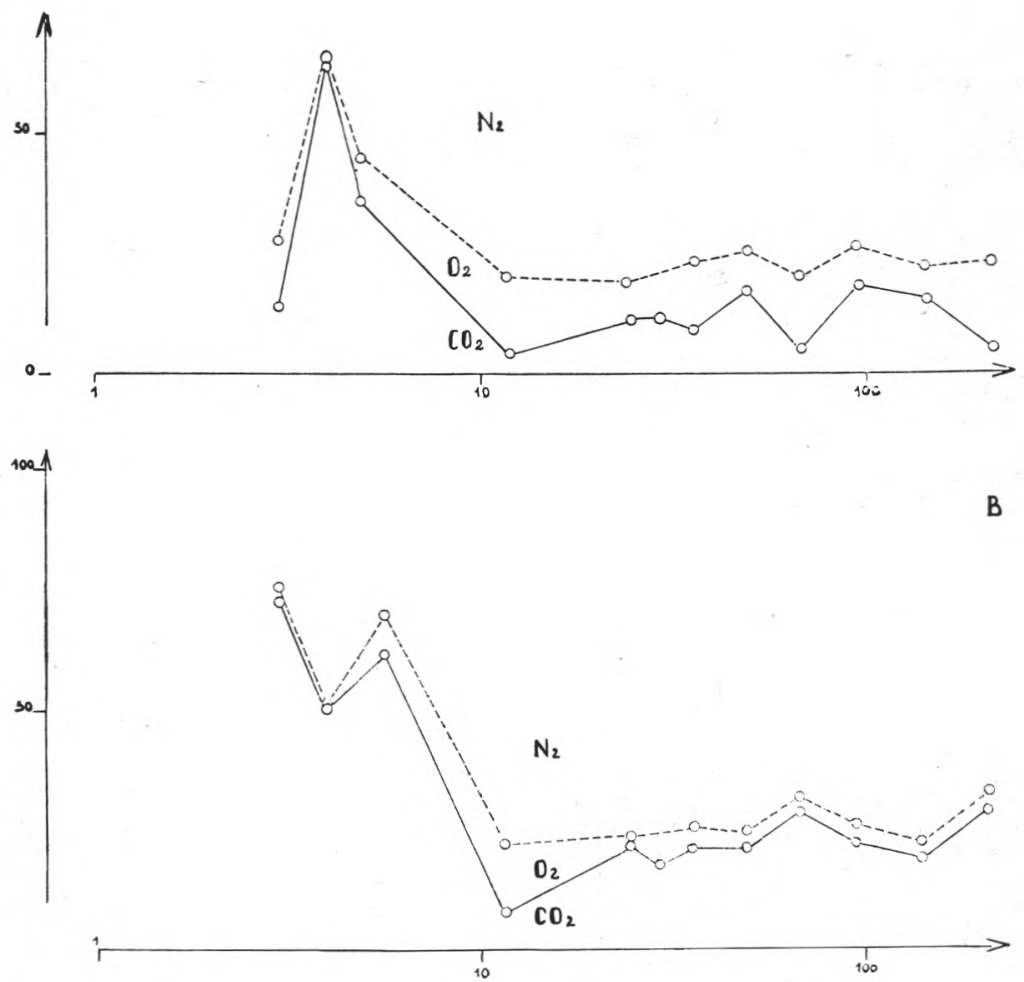

\section{Graphique $n^{\circ} 4$}

Composition de l'atmosphère du fromage
$\mathbf{A}=$ faces
$\mathrm{B}=$ centre.

Abscisses : temps exprimé en jours en coordonnées logarithmiques.

Ordonnées : azote, oxygène et gaz carbonique, en pourcentage du volume total. 


\section{III. - ETUDE DE LA FLORE DE LEVURE}

\section{a) Evolution de la flore totale}

L'évolution de la flore de levure au cours des diverses étapes de la fabrication a été suivie par des prélèvements au centre du fromage et en surface. Pour compléter ces observations, quelques prélèvements de révérum ont été aussi effectués.

L'utilisation de 8 milieux de culture différents pour tous les étalements permet de distinguer divers types de levures, en fonction de trois facteurs :

- Sensibilité au sel.

- Sensibilité aux pH faibles.

- Substrat de croissance.

En surface, la flore se développe rapidement jusqu'au salage. Les températures permettent jusque-là de différencier trois stades:

- Phase à $30^{\circ} \mathrm{C}$.

- Phase à $18^{\circ} \mathrm{C}$.

- Phase à $10^{\circ} \mathrm{C}$.

Le salage provoque une chute brutale; plus de 90 p. 100 de la flore disparaît. La croissance reprend ensuite très vite et la population maximum est atteinte au plombage (supérieure à $10^{\circ}$ ). Ensuite, pendant l'affinage sous feuille d'étain, la population diminue régulièrement (graphique $\mathrm{n}^{\circ} 5$ ).

Les populations obtenues en présence de glucose et d'acide lactique sont très voisines à tous les stades, aussi bien à $\mathrm{pH} 3$ qu'à $\mathrm{pH} 5$ (graphique $\mathrm{n}^{\circ} 5 \mathrm{C}$ et tableau $\mathrm{n}^{\circ} 2$ ). Toutes les levures présentes semblent donc donner une croissance aussi bonne sur acide lactique que sur glucose. Une importante flore sensible au sel (graphique $n^{\circ} 5$ B) est décelée avant salage. Elle ne disparaît que 10 jours environ après le salage. Il faut remarquer que de nombreuses levures ne poussent pas sur le milieu L 310 ; elles ne sont pas nécessairement très sensibles au sel car il semble que la conjonction des trois facteurs $(\mathrm{pH}$, sel et substrat) soit nécessaire.

Le milieu L 310 donne des populations beaucoup plus faibles que $G 310, L 30$ et L 510, alors que ces trois derniers donnent souvent des valeurs très voisines. Ce phénomène se retrouve à tous les stades de la fabrication et de l'affinage. Généralement, toutes les levures poussent indifféremment à $\mathrm{pH} 3$ et à $\mathrm{pH} 5$, sauf dans le prélèvement qui suit le salage (graphique $\mathrm{n}^{\circ} 5 \mathrm{~A}$ ). Il est possible que le sel ait provoqué soit une sensibilisation aux $\mathrm{pH}$ bas, des levures existantes, soit l'apparition éphémère d'une flore sensible aux $\mathrm{pH}$ bas.

Le révérum a donné des résultats analogues à ceux observés dans les prélèvements à la face du fromage. Les populations sont plus importantes puisqu'elles peuvent atteindre $3,10^{11}$ après plombage. La prolifération des levures qui a été constatée après le salage 


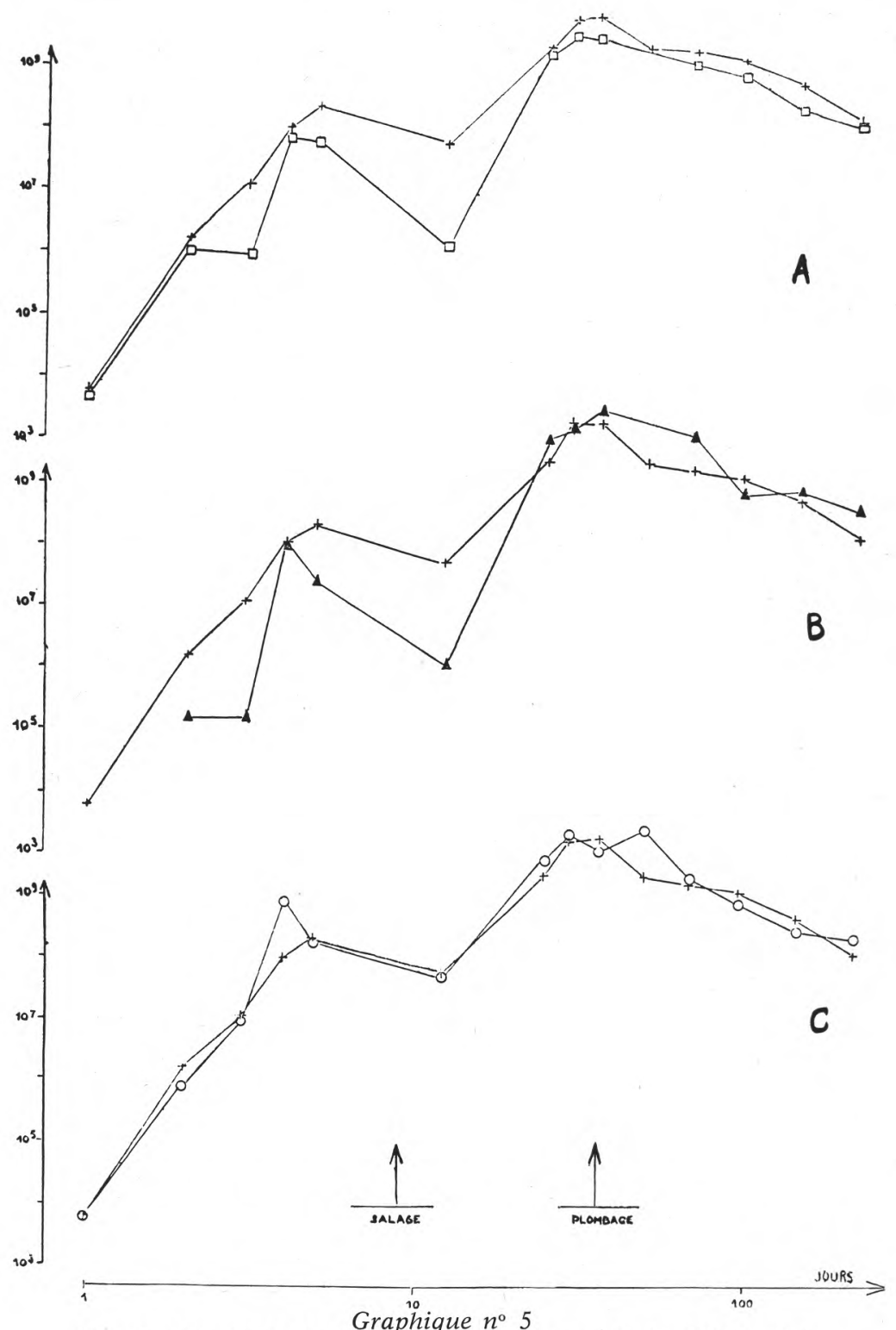

Numération de la flore de levures au cours de la fabrication à l'aide de plusieurs milieux d'étalement

(faces du fromage)

Coordonnées logarithmiques.

Abscisses : temps en jours.

Ordonnées : population exprimée en nombre de cellules par g de fromage.

+ milieu glucosé $\mathrm{pH}=5$

$\square$ milieu glucosé $\mathrm{pH}=3$

$\triangle$ milieu glucosé $\mathrm{pH}=5 \mathrm{ClNa}=10 \mathrm{p} \cdot 100$

milieu acide lactique $\mathrm{pH}=5$ 
$T A B L E A U n^{\circ} 2$. - Evolution de la flore totale de levures sur la face du fromage

(exprimée par le logarithme du nombre de cellules par g de fromage)

\begin{tabular}{|c|c|c|c|c|c|c|c|c|c|}
\hline \multicolumn{2}{|c|}{ Milieu d'étalement } & \multirow[b]{2}{*}{ G 50} & \multirow[b]{2}{*}{ G 510} & \multirow[b]{2}{*}{ G 30} & \multirow[b]{2}{*}{ G 310} & \multirow[b]{2}{*}{ L 50} & \multirow[b]{2}{*}{ L 510} & \multirow[b]{2}{*}{ L 30} & \multirow[b]{2}{*}{ L 310} \\
\hline Stades & $\begin{array}{l}\text { Temps } \\
\text { en } \\
\text { jours }\end{array}$ & & & & & & & & \\
\hline Lait & 1 & & & & & & & & \\
\hline Découpage & 1 & & & & & & & & \\
\hline $\begin{array}{l}\text { Mise en moule } \\
\text { et égouttage } \\
\text { du caillé }\end{array}$ & $\begin{array}{l}1 \\
2 \\
3\end{array}$ & $\begin{array}{l}3,8 \\
6,2 \\
7,1\end{array}$ & $\begin{array}{l}- \\
5,2 \\
5,2\end{array}$ & $\begin{array}{l}3,7 \\
6,0 \\
5,9\end{array}$ & $\begin{array}{l}3,6 \\
4,3 \\
5,2\end{array}$ & $\begin{array}{l}3,8 \\
5,9 \\
7,0\end{array}$ & $\begin{array}{l}3,8 \\
4,5 \\
5,7\end{array}$ & $\begin{array}{l}3,6 \\
5,7 \\
6,5\end{array}$ & $\begin{array}{l}- \\
3,0 \\
4,0\end{array}$ \\
\hline Fin d'égouttage & 4 & 8,0 & 8,0 & 7,8 & 5,8 & 8,9 & 8,3 & 7,5 & 4,0 \\
\hline Salle froide & 5 & 8,3 & 7,4 & 7,7 & 5,8 & 8,2 & 7,9 & 7,5 & 7,6 \\
\hline $\begin{array}{c}3 \text { jours après salage } \\
\text { et } \\
\text { premier affinage }\end{array}$ & $\begin{array}{l}12 \\
24 \\
29\end{array}$ & $\begin{array}{l}6,7 \\
9,3 \\
9,9\end{array}$ & $\begin{array}{l}6,1 \\
9,6 \\
9,8\end{array}$ & $\begin{array}{l}6,1 \\
9,2 \\
9,6\end{array}$ & $\begin{array}{l}6,2 \\
8,6 \\
7,7\end{array}$ & $\begin{array}{r}6,7 \\
9,6 \\
10,0\end{array}$ & $\begin{array}{l}6,5 \\
9,5 \\
9,6\end{array}$ & $\begin{array}{l}5,8 \\
9,1 \\
9,4\end{array}$ & $\begin{array}{l}5,4 \\
8,4 \\
6,8\end{array}$ \\
\hline $\begin{array}{c}\text { Plombage } \\
\text { et } \\
\text { deuxième } \\
\text { affinage }\end{array}$ & $\begin{array}{r}36 \\
50 \\
66 \\
95 \\
143 \\
216\end{array}$ & $\begin{array}{l}9,9 \\
9,5 \\
9,2 \\
9,1 \\
8,7 \\
8,1\end{array}$ & $\begin{array}{r}10,1 \\
9,7 \\
8,8 \\
8,9 \\
8,6\end{array}$ & $\begin{array}{l}9,5 \\
9,0 \\
8,8 \\
8,3 \\
8,0\end{array}$ & $\begin{array}{l}9,4 \\
9,1 \\
9,2 \\
8,9 \\
7,0 \\
8,1\end{array}$ & $\begin{array}{r}9,7 \\
11,1 \\
9,3 \\
8,9 \\
8,5 \\
8,3\end{array}$ & $\begin{array}{r}9,5 \\
11,1 \\
9,3 \\
9,0 \\
8,4 \\
8,2\end{array}$ & $\begin{array}{l}9,3 \\
8,9 \\
8,7 \\
8,5 \\
8,4 \\
7,6\end{array}$ & $\begin{array}{l}8,8 \\
8,0 \\
8,3 \\
8,4 \\
7,0 \\
5,9\end{array}$ \\
\hline
\end{tabular}


et avant le plombage est importante dans la conduite de l'affinage, sur deux plans : nuisible.

- Le révérun protège les faces contre l'implantation d'une flore

- Il permet au papier d'étain de coller parfaitement sur les faces au moment du plombage.

Cette prolifération assure de bonnes conditions d'affinage. La qualité du produit fini dépend en grande partie de la réussite de ces diverses opérations.

Au centre, la flore se développe rapidement jusqu'au salage. Le salage provoque un arrêt de la multiplication des levures, mais l'action du salage est beaucoup moins spectaculaire qu'en surface. En effet, le sel ne pénètre complètement jusqu'au centre qu'à partir du $10^{\text {me }}$ jour qui suit le salage. La population maximum est obtenue quelques jours après le plombage. Elle ne dépasse pas $10^{\circ}$ cellules au g et est donc toujours plus faible qu'en surface. La population diminue ensuite régulièrement pendant l'affinage sous feuille d'étain (graphique $n^{\circ} 6$ et tableau $n^{\circ} 3$ ).

De la fabrication au plombage, les numérations obtenues sur tous les milieux, à l'exception du L 310, donnent des résultats assez proches. Cependant, les prélèvements effectués en salle froide donnent des numérations sensiblement plus élevées sur le milieu glucosé à $\mathrm{pH} \mathrm{5,} \mathrm{sans} \mathrm{sel}(\mathrm{G} 50)$ que sur tous les autres milieux. Il se confirme que la présence simultanée d'un $\mathrm{pH} 3$, de 10 p. 100 de sel et d'acide lactique ne permet pas la croissance de nombreuses levures.

Pendant l'affinage sous étain, les numérations sur milieu G 50 donnent des valeurs supérieures à celles obtenues sur tous les autres milieux. Il semble qu'une flore sensible au sel (graphique $\mathrm{n}^{\circ} 6 \mathrm{~B}$ ), une flore sensible au $\mathrm{pH} 3$ (graphique $\mathrm{n}^{\circ} 6 \mathrm{~A}$ ) et une flore incapable de donner une croissance sur acide lactique (graphique $n^{\circ} 6 \mathrm{C}$ ) aient apparu. Le problème se pose de savoir si ces trois propriétés correspondent à un même type biologique ou à trois types différents.

Nous allons voir maintenant l'évolution de différents types biologiques au cours des processus de fabrication et d'affinage. Pour ce travail nous avons fait, sur tous les étalements, des prélèvements de tous les types coloniaux proportionnellement à la fréquence de chaque type. Des tests ont été effectués et ils ont permis une numération relative. Les résultats ainsi obtenus sont donnés dans les paragraphe $b$ ) et $c$ ), ils ont une valeur indicative qui permet de voir l'évolution des types dominants.

\section{b) Evolution des Endomycétacées}

Parmi les levures qui sporulent, le groupe assimilant le nitrate est, de beaucoup, le plus important. Certaines fermentent le glucose 


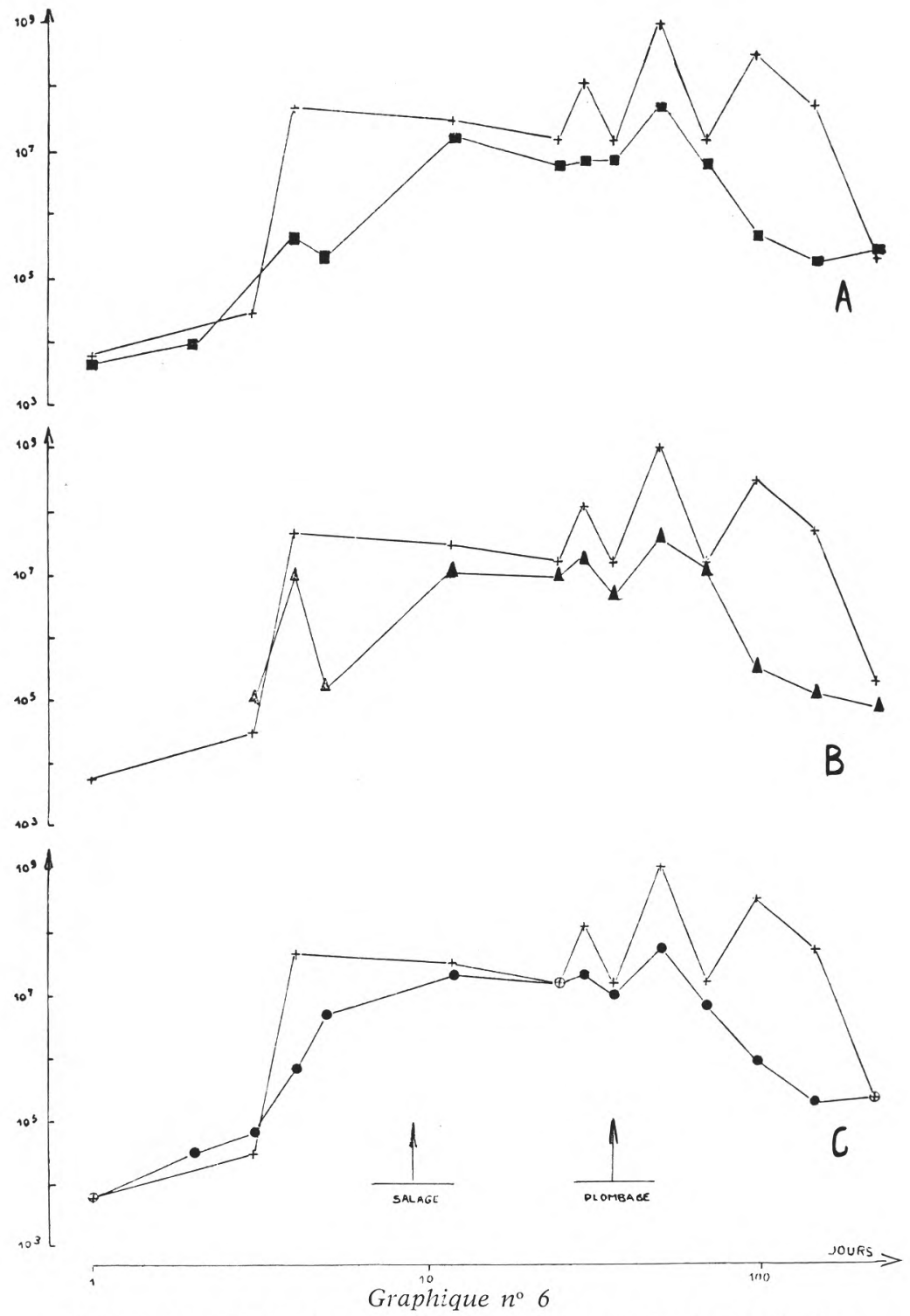

Numération de la flore de levures au cours de la fabrication à l'aide de plusieurs milieux d'étalement (centre du fromage)

Coordonnées logarithmiques.

Abscisses : temps en jours.

Ordonnées : population exprimée en nombre de cellules par g de fromage.

+ milieu glucosé $\mathrm{pH}=5$

$\square$ milieu glucosé $\mathrm{pH}=3$

$\triangle$ milieu glucosé $\mathrm{pH}=5 \quad \mathrm{CINa}=10 \mathrm{p} .100$

milieu acide lactique $\mathrm{pH}=5$ 
$T A B L E A U n^{\circ} 3$. - Evolution de la flore totale de levures dans le centre du fromage

(exprimée par le logarithme du nombre de cellules)

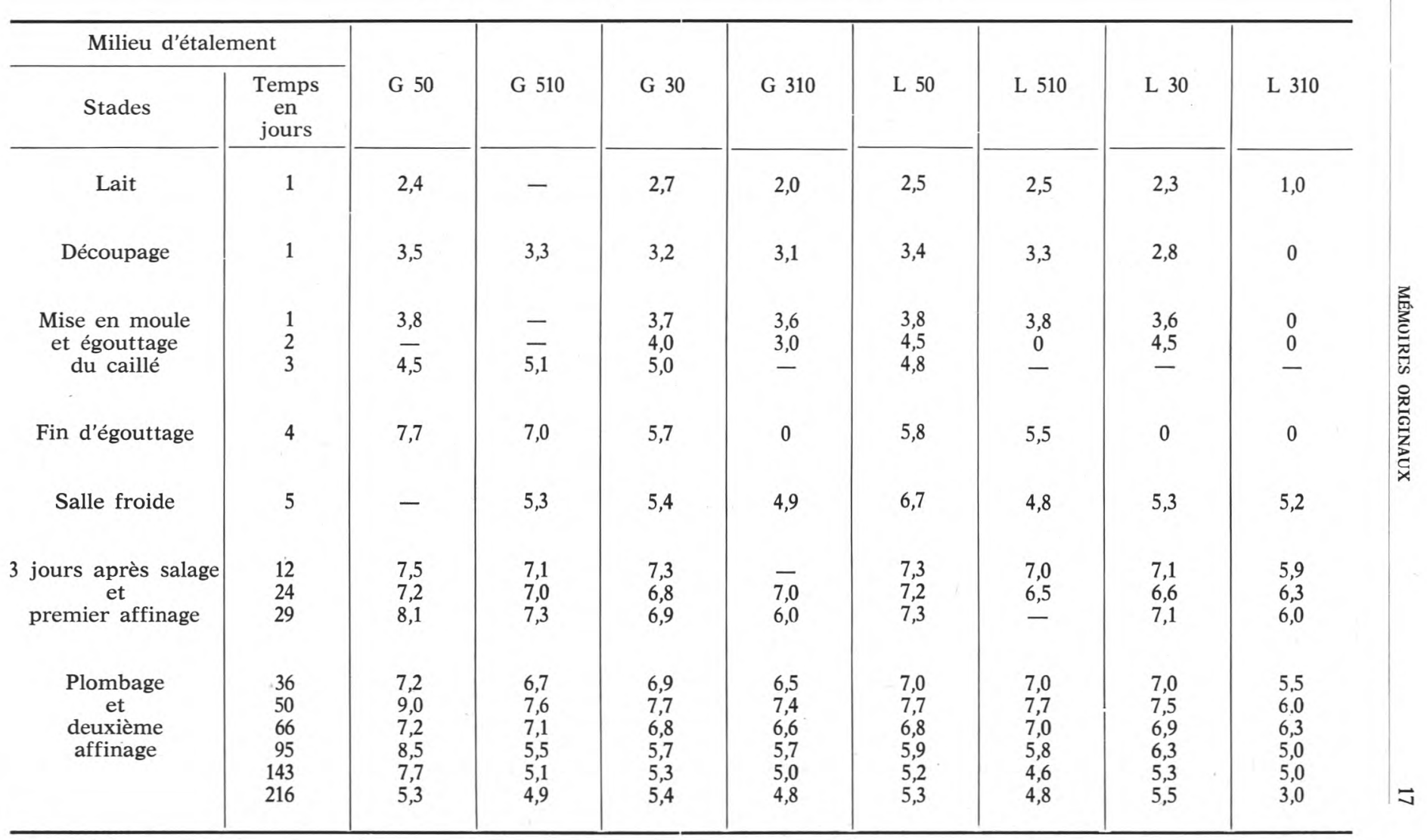


et appartiennent donc au genre Hansenula, H. et P. Sydow. Elles peuvent présenter un voile ou un anneau dans les cultures liquides et forment parfois un pseudomycélium. Certaines produisent des acides dans le milieu au carbonate de chaux de Lodder. Ces quatre caractères permettent de définir 9 types biologiques à eux seuls. Il est donc probable qu'un très grand nombre d'espèces pourra être défini dans ce groupe.

Certaines souches assimilent le nitrate et ne fermentent pas le glucose. Les types biologiques définis précédemment en fonction de la formation d'anneau et de voile, de la production d'acide en présence de carbonate de chaux et de la présence de pseudomycélium se retrouvent. Le genre Hansenula groupe en principe des souches qui fermentent le glucose. Les levures décrites ici. bien que ne fermentant pas, présentent des analogies extrêmement importantes avec les Hansenula du Roquefort et il est peut être possible d'envisager leur rattachement à ce genre.

Les ascosporées incapables d'assimiler le nitrate sont beaucoup moins nombreuses. Certaines fermentent le glucose et présentent les caractéristiques du genre Saccharomyces (Meyen) Reess. Elles ne forment pas de pseudomycélium. Certaines forment un anneau et, plus rarement, un léger voile. Toutes fermentent le lactose. Un sondage a permis de voir que la plupart de ces souches présentent les caractéristiques de l'espèce Saccharomyces lactis, Dombrowki. Des souches incapables d'assimiler le nitrate et de fermenter le glucose ont été également trouvées de façon épisodique. Elles ne présentent qu'un intérêt mineur et elles ne sont guère présentes que dans le lait. Elles forment un anneau, quelquefois un voile. Elles possèdent aussi parfois un pseudomycélium. Ces souches sont voisines du genre Debaryomyces, Lodder et van Rij.

Les graphiques $n^{\circ} 7$ et 8 représentent, respectivement pour les faces et le centre du fromage, l'évolution des populations de levures ascosporées correspondant aux quatre types :

- Nitrate + fermentation +
- Nitrate + fermentation -
- Nitrate - fermentation +
- Nitrate - fermentation -

Sur les faces du fromage, les souches du type nitrate - fermentation - ne sont présentes que de manière très épisodique et disparaissent complètement après le salage. Leur rôle, dans l'élaboration du fromage, doit être très peu important. Les Saccharomyces (nitrate - fermentation + ) sont plus importants ; ils se multiplient activement pendant la fabrication et peuvent représenter 1 à 8 p. 100 de la flore de levure avant le salage. Le salage élimine complètement toutes les levures nitrate - qui sporulent. A aucun moment, les levures qui ne réduisent pas les nitrates atteignent des populations comparables aux levures nitrate + . Parmi les sporulées, le groupe dominant est toujours constitué par des levures qui assimilent le 

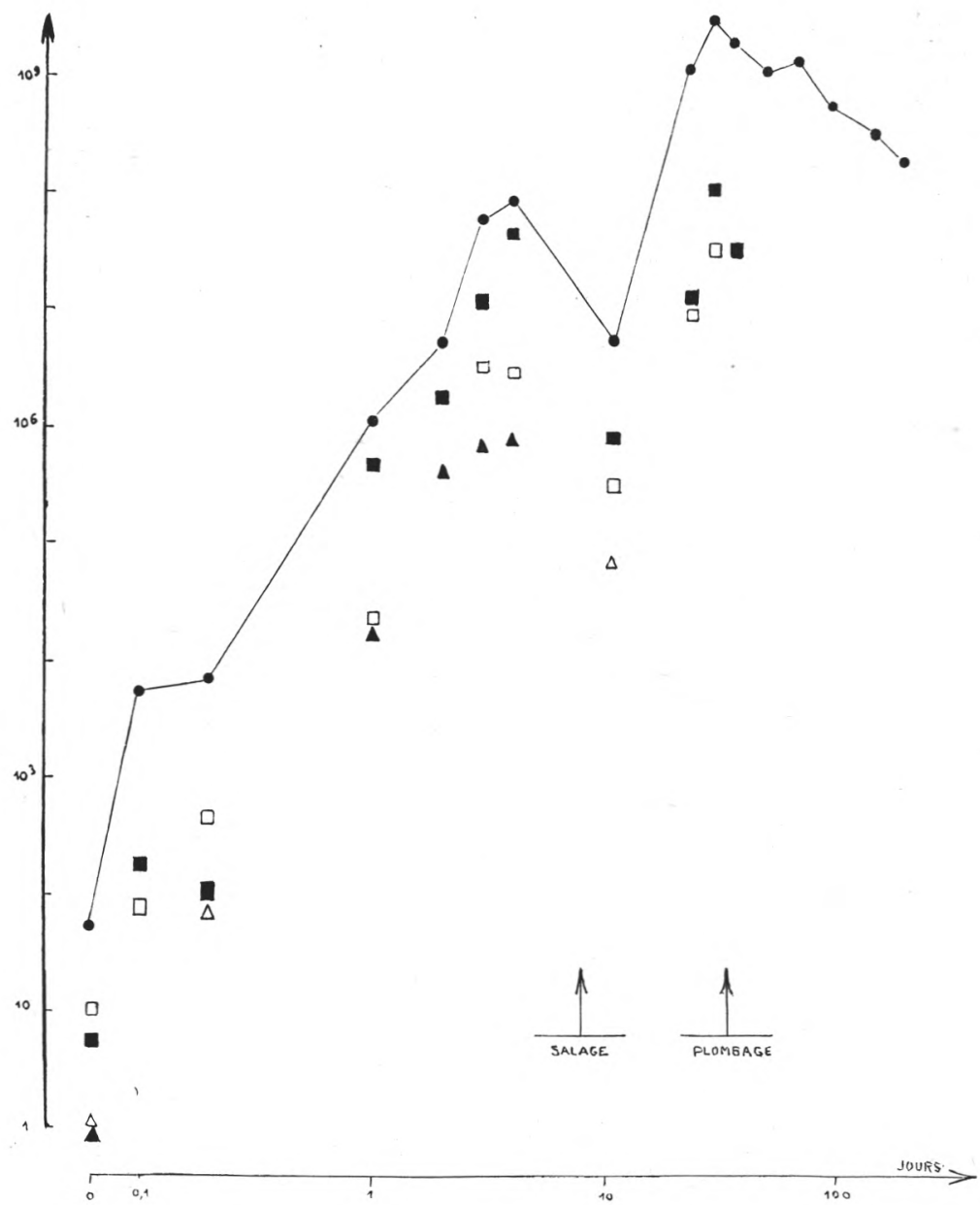

Graphique $n^{\circ} 7$

Evolution de la flore de levures de la famille des Endomycétacées (faces du fromage)

Coordonnées logarithmiques.

Abscisses : temps en jours.

Ordonnées : populations exprimées en nombre de cellules par g de fromage.

- flore totale

- nitrate + fermentation +

$\square$ nitrate + fermentation -

A nitrate - fermentation +

$\triangle$ nitrate - fermentation - 

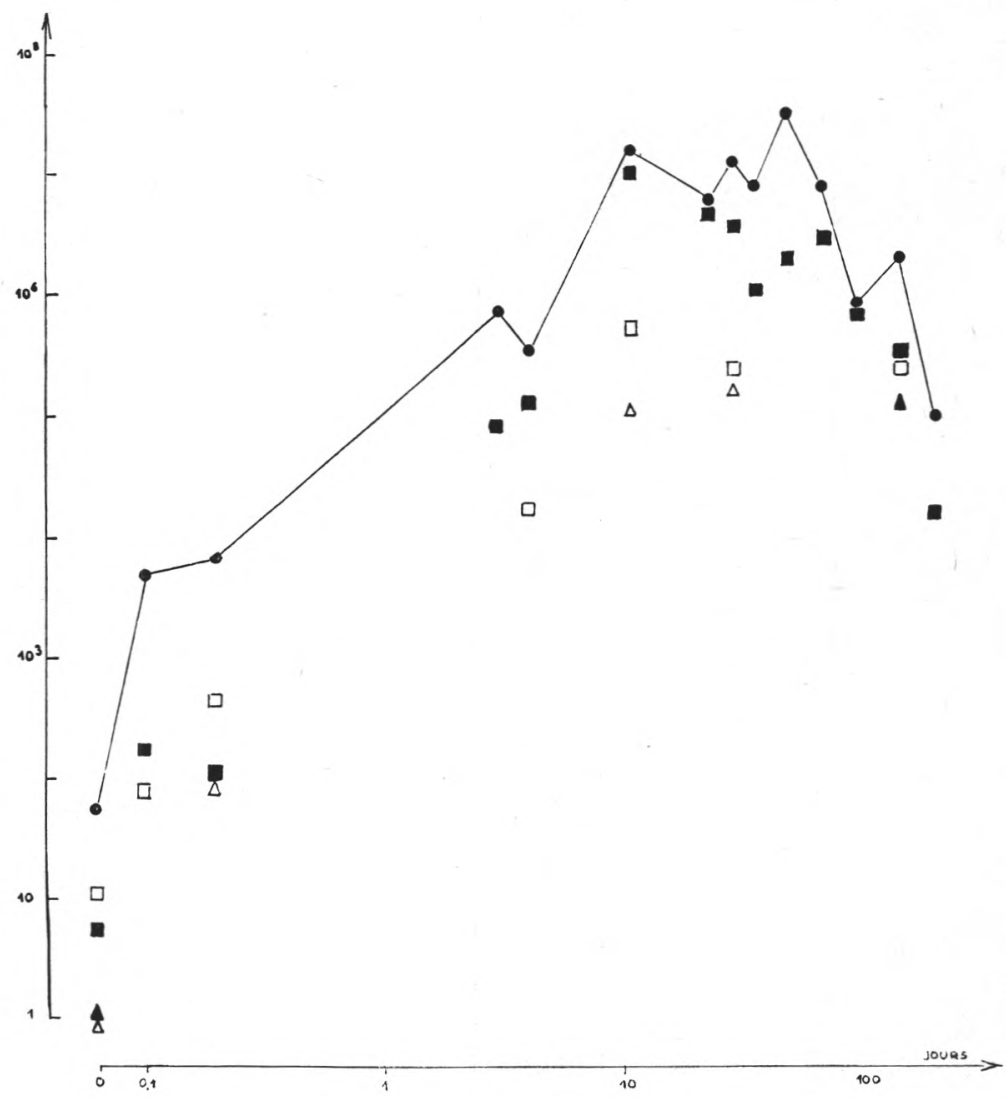

\section{Graphique $n^{\circ} 8$}

Evolution de la flore de levures de la famille des Endomycétacées (centre du fromage)

Coordonnées logarithmiques.

Abscisses : temps en jours.

Ordonnées : populations exprimées en nombre de cellules par g de fromage.

- flore totale

nitrate + fermentation +

$\square$ nitrate + fermentation -

A nitrate - fermentation +

$\triangle$ nitrate - fermentation - 
nitrate, fermentent le glucose et présentent les caractères généraux des Hansenula. Leur nombre diminue au salage comme d'ailleurs celui de la flore totale, mais leur multiplication reprend ensuite très rapidement. Elles ne disparaissent qu'au plombage. Les levures qui réduisent les nitrates mais ne fermentent pas le glucose suivent une évolution analogue, se multiplient rapidement, diminuent de nombre au salage, se multiplient ensuite pendant le premier affinage et disparaissent après le plombage. Leur nombre est toujours très inférieur à celui des Hansenula typiques. En définitive, les levures nitrate + sont plus nombreuses que les nitrates - ; ces dernières disparaissent plus tôt, c'est-à-dire au salage. Dans ces deux catégories, les levures à métabolisme fermentaire sont nettement plus nombreuses que les levures à métabolisme exclusivement oxydatif (graphique $\mathrm{n}^{\circ} 7$ ).

$\mathrm{Au}$ centre du fromage, les levures de la famille des Endomycétacées sont en général capables de réduire le nitrate. A tous les stades, des Hansenula capables d'assimiler les nitrates et de fermenter le glucose sont présents en abondance. Ces souches représentent 88 p. 100 de la flore de levure, trois jours après salage, et en représentent encore 16 p. 100 à la vente. Les souches capables d'assimiler le nitrate, mais incapables de fermenter, sont en général présentes mais elles n'ont pas été détectées dans certains prélèvements. Elles sont moins nombreuses et moins importantes que les Hansenula typiques. Les levures qui n'assimilent pas le nitrate (Saccharomyces et Debaryomyces) n'ont été trouvées que de façon très épisodique. Ce n'est qu'exceptionnellement qu'elles représentent plus de 2 ou 3 p. 100 de la flore de levure. Le salage n'a que peu marqué au centre du fromage. Il en résulte que la flore est sensiblement la même après et avant le salage (graphique $n^{\circ} 8$ ).

\section{c) Evolution des Cryptococcacées}

Les levures anascosporées trouvées dans ce cuvier appartiennent à trois genres :

- Torulopsis Berlese.

- Candida Berkhout.

- Rhodotorula Harrison.

Les Torulopsis constituent le groupe le plus important. Elles se retrouvent tout au long de la fabrication et de l'affinage. Ces souches n'ont pratiquement ni mycélium, ni pseudomycélium. Elles forment souvent des anneaux et parfois des voiles. Certaines donnent une acidification sur le milieu contenant du carbonate de chaux. En outre, comme pour les ascosporées, il est possible de trouver les quatre types biologiques :

- Nitrate + fermentation +

- Nitrate + fermentation -

- Nitrate - fermentation +

- Nitrate - fermentation - 
Ces divers caractères nous ont permis de classer ces souches en 19 types biologiques. Un très grand nombre d'espèces de Torulopsis était donc certainement présent dans ces fromages.

Les Candida sont moins nombreux et présents seulement dans le lait et dans les premiers stades de la fabrication. Ils ont des pseudomycéliums ou des mycéliums, forment presque toujours des anneaux et très souvent des voiles. Certains donnent des acides dans le milieu au carbonate de calcium. Ici encore on retrouve les quatre types :

$$
\begin{aligned}
& \text { - Nitrate + fermentation + } \\
& \text { - Nitrate + fermentation - } \\
& \text { - Nitrate - fermentation + } \\
& \text { - Nitrate - fermentation - }
\end{aligned}
$$

Ces quelques caractères ont permis de classer ces souches en 18 groupes biologiques. Bien que peu nombreux, les Candida sont donc représentés par de nombreuses espèces.

Les souches présentant une pigmentation rouge comme les Rhodotorula sont peu nombreuses et ne se trouvent pratiquement que dans le lait et les premiers stades de la fabrication. Elles ne donnent que très rarement des pseudomycélium mais forment le plus souvent des anneaux et parfois des voiles. Certaines assimilent le nitrate et fermentent le glucose, d'autres assimilent le nitrate et ne fermentent pas le glucose, d'autres enfin n'assimilent pas le nitrate et ne fermentent pas le glucose. Aucune souche incapable d'assimiler le nitrate ne fermente le glucose. Parmi ces souches présentant la pigmentation typique des Rhodotorula, il est curieux de constater que certaines donnent une fermentation, ce qui est inhabituel chez ce genre. La présence de voile ne correspond pas non plus au type général du genre. Au cours de ce travail, de nombreux types biologiques inhabituels ont été trouvés. Leur étude taxonomique sera faite ultérieurement. Dans cette étude, nous nous sommes volontairement limités à des critères présentant un intérêt technologique.

Sur les graphiques $\mathrm{n}^{\text {os }} 9$ et 10 , l'évolution de la flore de Torulopsis au cours de la fabrication et de l'affinage est suivie. Sont représentés, séparément, les quatre types biologiques :

- Nitrate + fermentation +

- Nitrate + fermentation -

- Nitrate - fermentation +

- Nitrate - fermentation -

Il nous a paru nécessaire de les distinguer car ces quatre types se retrouvent chez les ascosporées d'une part, et dans tous les genres d'anascosporées, à l'exception des Rhodotorula, d'autre part.

Sur les faces du fromage, la multiplication des Torulopsis est active au début de la fabrication. Le groupe incapable de fermenter le glucose et d'assimiler les nitrates est souvent absent et représente rarement une fraction importante de la flore. Les trois autres 


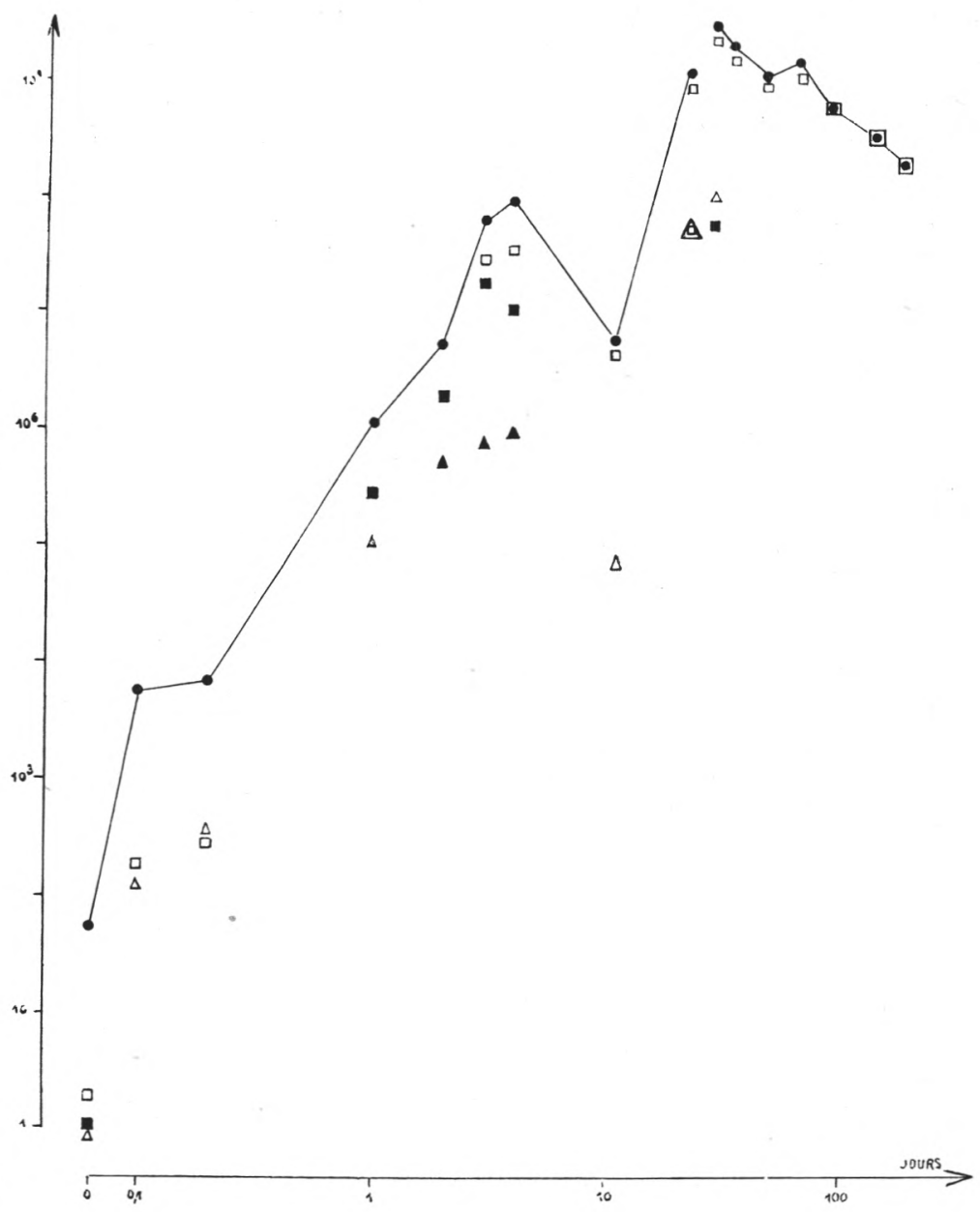

Graphique $n^{\circ} 9$

Evolution de la flore de levures de la famille des Cryptococcacées (faces du fromage)

Cordonnées logarithmiques.

Abscisses : temps en jours.

Ordonnées : populations exprimées en nombre de cellules par $\mathrm{g}$ de fromage.

- flore totale

nitrate + fermentation +

$\square$ nitrate + fermentation -

$\Delta$ nitrate - fermentation +

$\triangle$ nitrate - fermentation - 


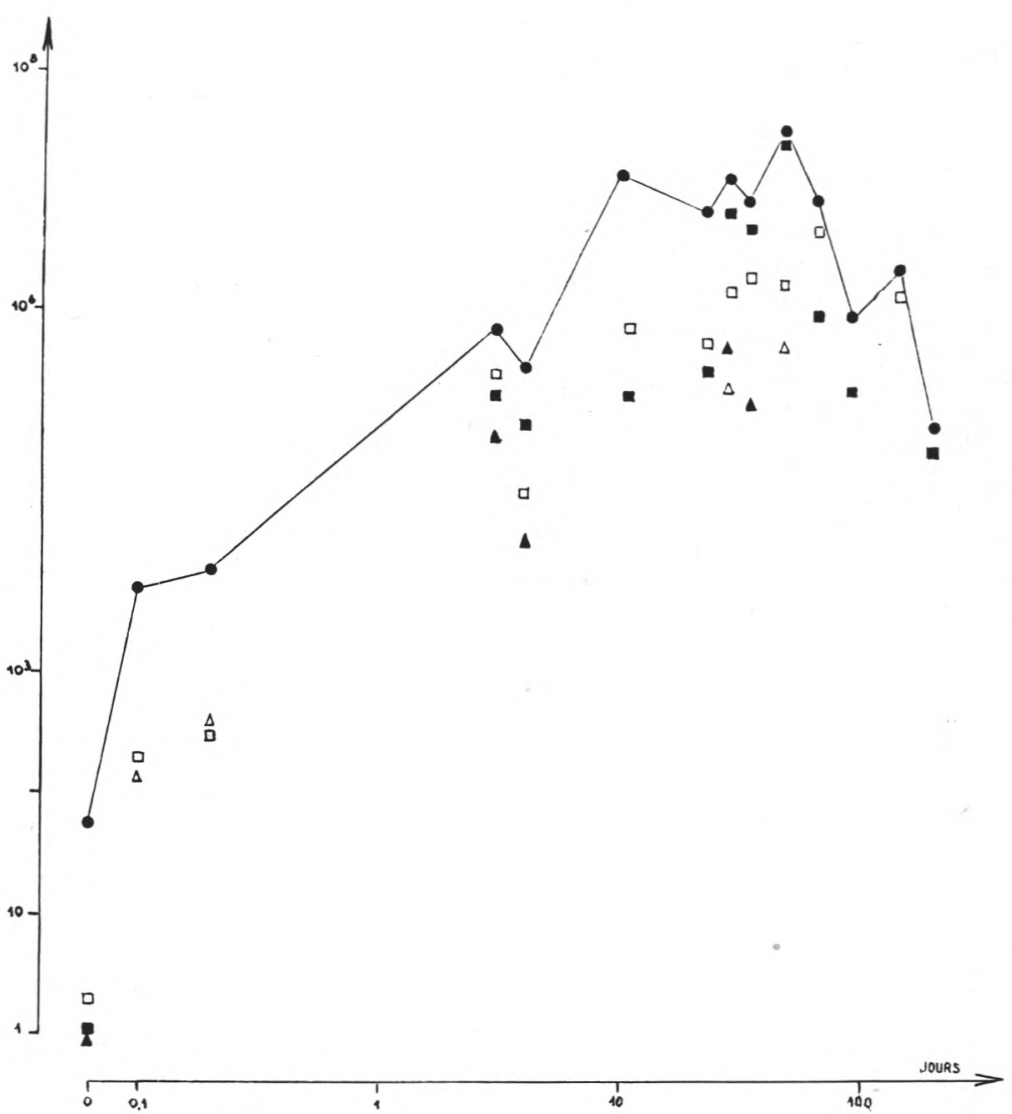

Graphique $n^{\circ} 10$

Evolution de la flore de levures de la famille des Cryptococcacées (centre du fromage)

Coordonnées logarithmiques.

Abscisses : temps en jours.

Ordonnées : populations exprimées en nombre de cellules par g de fromage.

- flore totale

- nitrate + fermentation +

$\square$ nitrate + fermentation -

$\Delta$ nitrate - fermentation +

$\triangle$ nitrate - fermentation - 
groupes sont en général présents. Les souches assimilant le nitrate représentent la flore dominante. Au salage, la population de Torulopsis baisse. La multiplication reprend ensuite très activement pendant le premier affinage pour atteindre un maximum le $100^{\text {me }}$ jour. La flore dominante est constituée alors par des Torulopsis qui ne fermentent pas le glucose et assimilent les nitrates. Après le plombage les Torulopsis de ce groupe constituent pratiquement la totalité de la population.

Au centre, l'action du salage est peu nette, comme nous l'avons déjà maintes fois constaté. Il en résulte que la flore reste plus variée après le salage qu'en surface. Les quatre types de Torulopsis peuvent se trouver, ensemble ou séparément, à tous les stades jusqu'à la vente. Il est possible que la flore ne soit pas identique, dans le détail de sa composition, pour tous les fromages. Cependant les Torulopsis qui assimilent le nitrate sont toujours les plus nombreux. Les Torulopsis incapables d'assimiler le nitrate deviennent très rares à partir du plombage. Au centre du fromage, parmi les souches assimilant le nitrate, celles qui fermentent le glucose sont les plus abondantes alors que l'inverse est observé en surface.

\section{d) Levures productrices d'acides}

Les levures acidogènes ont été prélevées dans les divers étalements. Elles sont présentes systématiquement à divers stades de la fabrication. Leur importance dans la flore du Roquefort a été étudiée. Il a été distingué, pour présenter les résultats, les levures acidogènes qui fermentent le glucose et les levures acidogènes à métabolisme exclusivement oxydatif.

Le graphique $n^{\circ} 11$ donne, pour la face des fromages, l'évolution de la flore totale et de la flore de levures productrices d'acide. A certains stades il ne figure pas de points représentatifs de la flore acidogène ; cela correspond à des prélèvements où les levures productrices-d'acides représentaient moins de 2 p. 100 de la flore de levure totale. Ces cas sont assez nombreux. Il semble cependant que les levures acidogènes sont importantes car elles peuvent atteindre par exemple 30 p. 100 de la flore de levure pendant l'égouttage. Au début de la fabrication, ces levures se multiplient très rapidement, comme d'ailleurs la population totale de levure. Les levures acidogènes disparaissent au salage ; il semble qu'elles soient particulièrement sensibles à l'action du sel et à l'élévation de l'extrait sec. Elles réapparaissent ensuite pendant le premier affinage pour disparaître à nouveau après le plombage. Des souches acidogènes se trouvent parmi tous les groupes mis en évidence. La flore acidogène est très variée au début de la fabrication. Mais, pendant le premier affinage, toutes les levures acidogènes appartiennent au genre Torulopsis. Le salage a donc éliminé de nombreuses espèces.

$\mathrm{Au}$ centre du fromage, les populations de levures sont plus faibles (graphique $\mathrm{n}^{\circ} 12$ ). Mais, la flore acidogène occupe une place 


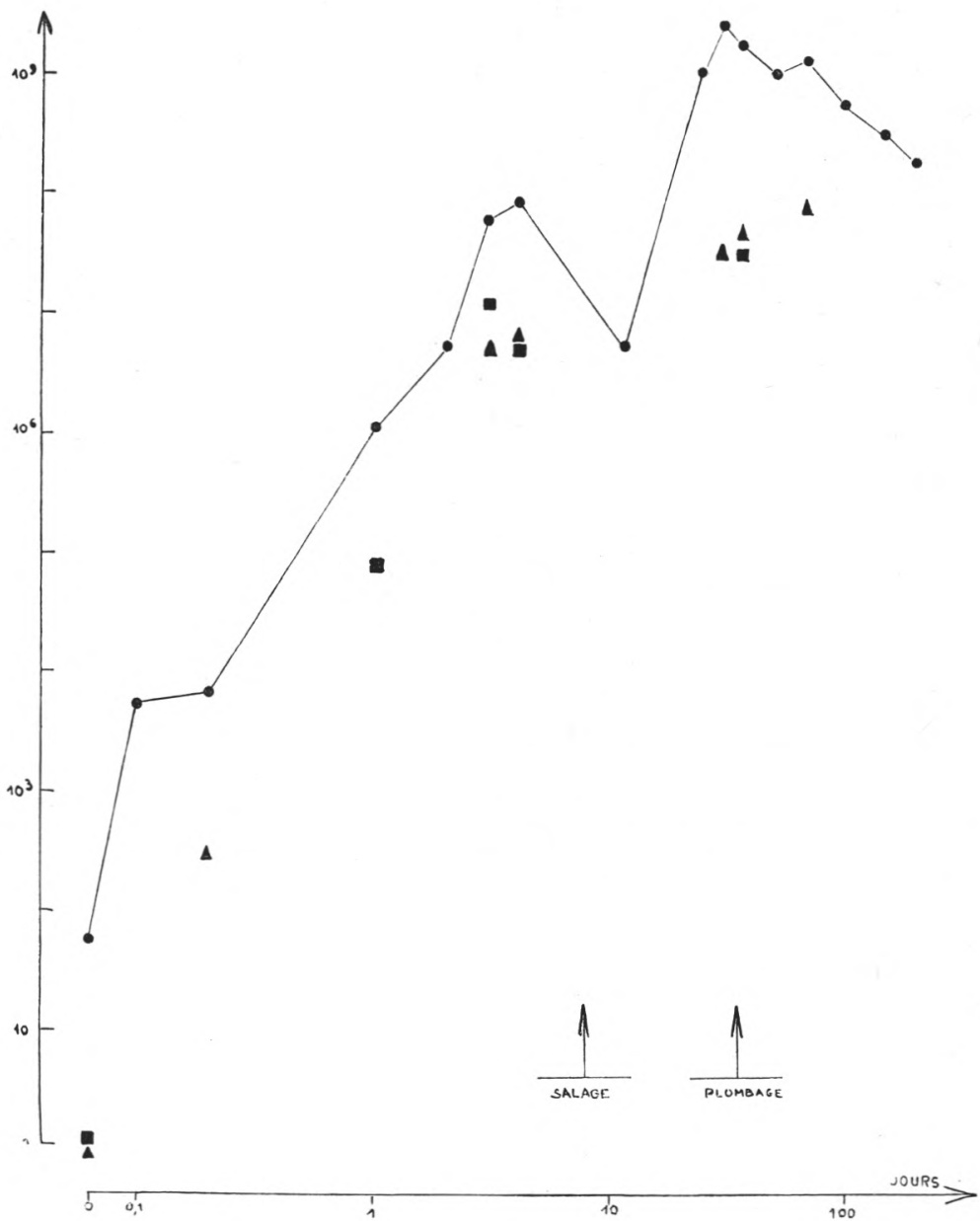

Graphique $n^{\circ} 11$

Evolution de la flore de levures acidogènes (faces du fromage)

Coordonnées logarithmiques.

Abscisses : temps en jours.

Ordonnées : populations exprimées en nombre de cellules par g de fromage

- flore totale de levure

- levures acidifiantes à métabolisme fermentaire

A levures acidifiantes à métabolisme exclusivement oxydatif 


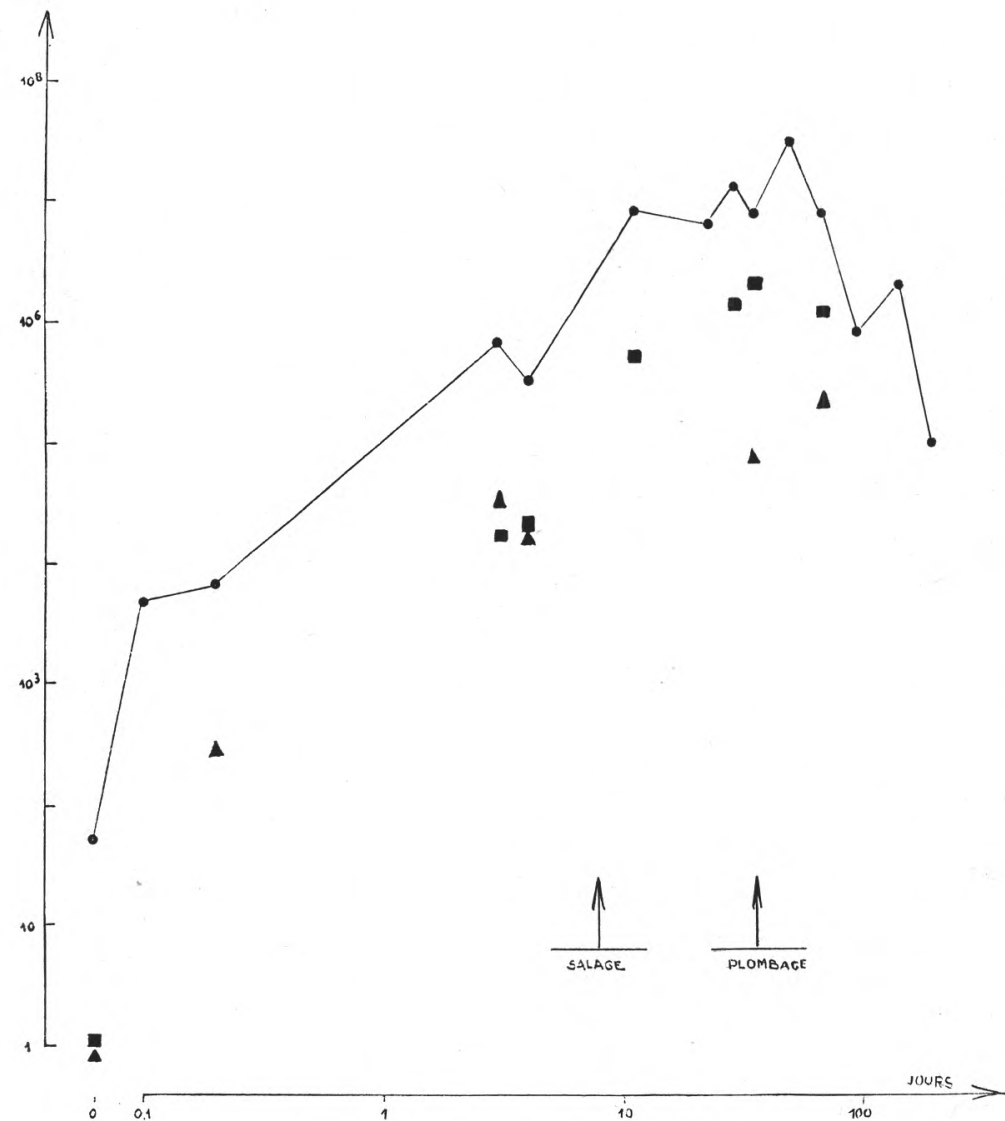

Graphique $n^{\circ} 12$

Evolution de la flore de levures acidogènes (centre du fromage)

Coordonnées logarithmiques.

Abscisses : temps en jours.

Ordonnées : populations exprimées en nombre de cellules par $\mathrm{g}$ de fromage

- flore totale de levure

- levures acidifiantes à métabolisme fermentaire

A levures acidifiantes à métabolisme exclusivement oxydatif 
importante. Parmi ces souches acidogènes, celles qui sont capables de fermenter le glucose sont beaucoup plus nombreuses que celles qui ont uniquement un métabolisme oxydatif. Il n'en est pas ainsi sur les faces du fromage. Le salage a peu marqué dans l'évolution de la flore au centre du fromage. Il en résulte que les souches acidogènes se maintiennent plus régulièrement qu'en surface. Elles ne disparaissent qu'un mois après le plombage. Jusqu'à ce moment, la flore acidogène est très variée : Torulopsis, Hansenula, etc. La disparition de certains types biologiques observée sur les faces du fromage après le salage ne se produit pas.

\section{DISCUSSION}

L'étude taxonomique des souches isolées devra être réalisée ; elle peut présenter un grand intérêt par la variété des types biologiques mis en évidence. Deux observations au moins ont été faites. Il existe d'abord dans le Roquefort une flore importante de levure assimilant le nitrate et présentant les principaux caractères du genre Hansenula H. et P. Sydow ; mais une partie de ces souches n'a pas la possibilité de fermenter les glucides. Il est possible qu'il soit nécessaire de modifier la définition du genre Hansenula qui est, en principe, constitué de souches fermentant vigoureusement les glucides. L'autre remarque est la mise en évidence d'une flore extrêmement variée présentant des colonies pigmentées en rouge comme les Rhodotorula. Il n'est pas certain que toutes ces souches soient des Rhodotorula Harrison et l'étude de la constitution de ces pigments devra être réalisée. [De toute manière, il y a là un travail intéressant qui devra être poursuivi ultérieurement].

Les types biologiques mis en évidence dans les levures du Roquefort sont nombreux. Mais, certaines caractéristiques se retrouvent régulièrement dans la flore. Le tableau $n^{\circ} 4$ indique, pour les faces du fromage, les pourcentages de sporulées et d'anascosporées, les pourcentages de souches assimilant le nitrate ou non, les pourcentages de levures à métabolisme fermentaire ou à métabolisme exclusivement oxydatif, et les pourcentages de levures acidogènes et de levures ne donnant pas d'acidité dans le milieu. Ce tableau donne une image de l'importance relative de certains types physiologiques aux diverses étapes de la préparation du fromage. Les mêmes données sont groupées dans le tableau $n^{\circ} 5$, pour le centre du fromage.

Le tableau $n^{\circ} 6$ donne, pour les faces et le centre, l'évolution de l'importance relative des levures et des microcoques dans la flore. Il indique les valeurs des rapports microcoques/levures, levures/flore totale et flore acidifiante/flore totale. L'étude de ces tableaux, complétant les graphiques 7 à 12, permet de définir l'évolution de la flore au cours des diverses étapes de la fabrication.

Dans le lait, la flore est très variée. Les levures sont très peu nombreuses, mais tous les types physiologiques sont représentés. 
TABLEAU n' 4. - Fréquence dans la flore de divers caractères taxonomiques ou technologiques (en p. 100) (faces du fromage)

\begin{tabular}{|c|c|c|c|c|c|c|c|c|c|}
\hline Stades & $\begin{array}{l}\text { Temps } \\
\text { en } \\
\text { jours }\end{array}$ & Sporulé & $\begin{array}{c}\text { Non } \\
\text { sporulé }\end{array}$ & $\begin{array}{c}\text { Nitrate } \\
+\end{array}$ & $\begin{array}{c}\text { Nitrate } \\
-\end{array}$ & $\begin{array}{l}\text { Fermen- } \\
\text { tation + }\end{array}$ & $\begin{array}{l}\text { Fermen- } \\
\text { tation - }\end{array}$ & $\begin{array}{c}\text { Acidifi- } \\
\text { cation + }\end{array}$ & $\begin{array}{l}\text { Acidifi- } \\
\text { cation - }\end{array}$ \\
\hline Lait & 0 & 33 & 67 & 67 & 33 & 17 & 83 & 4 & 96 \\
\hline Découpage & 0 & 5 & 95 & 78 & 22 & 30 & 70 & 0 & 100 \\
\hline $\begin{array}{l}\text { Mise au moule } \\
\text { et égouttage } \\
\text { du caillé }\end{array}$ & $\begin{array}{l}0 \\
1 \\
2\end{array}$ & $\begin{array}{r}9 \\
46 \\
41\end{array}$ & $\begin{array}{l}91 \\
54 \\
59\end{array}$ & $\begin{array}{l}89 \\
87 \\
82\end{array}$ & $\begin{array}{l}11 \\
13 \\
18\end{array}$ & $\begin{array}{r}32 \\
98 \\
100\end{array}$ & $\begin{array}{r}68 \\
2 \\
0\end{array}$ & $\begin{array}{r}5 \\
77 \\
0\end{array}$ & $\begin{array}{r}95 \\
23 \\
100\end{array}$ \\
\hline Egouttage & 3 & 27 & 73 & 97 & 3 & 49 & 51 & 29 & 71 \\
\hline Salle froide & 4 & 54 & 46 & 98 & 2 & 61 & 39 & 12 & 88 \\
\hline $\begin{array}{c}3 \text { jours après salage } \\
\text { et } \\
\text { premier affinage }\end{array}$ & $\begin{array}{l}11 \\
23 \\
28\end{array}$ & $\begin{array}{r}20 \\
2 \\
5\end{array}$ & $\begin{array}{l}80 \\
98 \\
95\end{array}$ & $\begin{array}{l}98 \\
96 \\
96\end{array}$ & $\begin{array}{l}2 \\
4 \\
4\end{array}$ & $\begin{array}{r}15 \\
10 \\
5\end{array}$ & $\begin{array}{l}85 \\
90 \\
95\end{array}$ & $\begin{array}{l}0 \\
0 \\
1\end{array}$ & $\begin{array}{r}100 \\
100 \\
99\end{array}$ \\
\hline $\begin{array}{c}\text { Plombage } \\
\text { et } \\
\text { deuxième } \\
\text { affinage }\end{array}$ & $\begin{array}{r}35 \\
49 \\
65 \\
94 \\
142\end{array}$ & $\begin{array}{l}2 \\
0 \\
0 \\
0 \\
0\end{array}$ & $\begin{array}{r}98 \\
100 \\
100 \\
100 \\
100\end{array}$ & $\begin{array}{r}100 \\
100 \\
97 \\
100 \\
100\end{array}$ & $\begin{array}{l}0 \\
0 \\
3 \\
0 \\
0\end{array}$ & $\begin{array}{l}2 \\
0 \\
0 \\
0 \\
0\end{array}$ & $\begin{array}{r}98 \\
100 \\
100 \\
100 \\
100\end{array}$ & $\begin{array}{l}5 \\
0 \\
6 \\
0 \\
0\end{array}$ & $\begin{array}{r}95 \\
100 \\
94 \\
100 \\
100\end{array}$ \\
\hline Vente & 197 & 0 & 100 & 100 & 0 & 0 & 100 & 0 & 100 \\
\hline
\end{tabular}


TABLEAU $N^{\circ}$ 5. - Fréquence dans la flore de divers caractères taxonomiques ou technologiques (en p. 100) (centre du fromage)

\begin{tabular}{|c|c|c|c|c|c|c|c|c|c|}
\hline Stades & $\begin{array}{c}\text { Temps } \\
\text { en } \\
\text { jours }\end{array}$ & Sporulé & $\begin{array}{l}\text { Non } \\
\text { sporulé }\end{array}$ & $\begin{array}{c}\text { Nitrate } \\
+\end{array}$ & $\begin{array}{c}\text { Nitrate } \\
-\end{array}$ & $\begin{array}{l}\text { Fermen- } \\
\text { tation }+\end{array}$ & $\begin{array}{l}\text { Fermen- } \\
\text { tation - }\end{array}$ & $\begin{array}{c}\text { Acidifi- } \\
\text { cation + }\end{array}$ & $\begin{array}{l}\text { Acidifi- } \\
\text { cation - }\end{array}$ \\
\hline Lait & 0 & 33 & 67 & 67 & 33 & 17 & 83 & 4 & 96 \\
\hline Découpage & 0 & 5 & 95 & 78 & 22 & 30 & 70 & 0 & 100 \\
\hline Moulage & 0 & 9 & 91 & 89 & 11 & 32 & 68 & 5 & 95 \\
\hline Fin d'égouttage & 3 & 12 & 88 & 88 & 12 & 49 & 51 & 7 & 93 \\
\hline Fin de salle froide & 4 & 39 & 61 & 97 & 3 & 79 & 21 & 11 & 89 \\
\hline $\begin{array}{l}3 \text { jours après salage } \\
\text { et premier affinage }\end{array}$ & $\begin{array}{l}11 \\
23\end{array}$ & $\begin{array}{l}92 \\
74\end{array}$ & $\begin{array}{r}8 \\
26\end{array}$ & $\begin{array}{r}99 \\
100\end{array}$ & $\begin{array}{l}1 \\
0\end{array}$ & $\begin{array}{l}91 \\
90\end{array}$ & $\begin{array}{r}9 \\
10\end{array}$ & $\begin{array}{l}3 \\
0\end{array}$ & $\begin{array}{r}97 \\
100\end{array}$ \\
\hline Premier affinage & 28 & 32 & 68 & 93 & 7 & 83 & 17 & 7 & 93 \\
\hline $\begin{array}{c}\text { Plombage } \\
\text { et } \\
\text { deuxième } \\
\text { affinage }\end{array}$ & $\begin{array}{r}35 \\
49 \\
65 \\
94 \\
142\end{array}$ & $\begin{array}{l}13 \\
57 \\
34 \\
77 \\
33\end{array}$ & $\begin{array}{l}87 \\
43 \\
66 \\
23 \\
67\end{array}$ & $\begin{array}{r}98 \\
99 \\
100 \\
100 \\
33\end{array}$ & $\begin{array}{r}2 \\
1 \\
0 \\
0 \\
67\end{array}$ & $\begin{array}{r}74 \\
96 \\
45 \\
100 \\
20\end{array}$ & $\begin{array}{r}26 \\
4 \\
55 \\
0 \\
80\end{array}$ & $\begin{array}{r}28 \\
0 \\
17 \\
0 \\
0\end{array}$ & $\begin{array}{r}72 \\
100 \\
83 \\
100 \\
100\end{array}$ \\
\hline Vente & 197 & 16 & 84 & 100 & 0 & 100 & 0 & 0 & 100 \\
\hline
\end{tabular}


TABLEAU No 6. - Evolution relative des constituants de la flore totale

\begin{tabular}{|c|c|c|c|c|c|c|c|}
\hline \multirow{2}{*}{ Stades } & \multirow{2}{*}{ Jours } & \multicolumn{2}{|c|}{\begin{tabular}{|c|} 
Rapport flore acidifiante/ \\
flore totale
\end{tabular}} & \multicolumn{2}{|c|}{$\begin{array}{l}\text { Rapport } \\
\text { levures/flore totale }\end{array}$} & \multicolumn{2}{|c|}{$\begin{array}{c}\text { Rapport } \\
\text { microcoques/levures }\end{array}$} \\
\hline & & face & centre & face & centre & face & centre \\
\hline Lait & 0 & 0,97 & 0,97 & 0,000005 & 0,000005 & 2280 & 2280 \\
\hline Découpage & 0 & 0,97 & 0,97 & 0,00007 & 0,00007 & 18 & 18 \\
\hline Moulage & 0 & 0,93 & 0,93 & 0,00001 & 0,00001 & 143 & 143 \\
\hline Fromage & $\begin{array}{l}1 \\
2\end{array}$ & $\begin{array}{l}1,00 \\
0,99\end{array}$ & $\begin{array}{l}1,00 \\
0,98\end{array}$ & $\begin{array}{l}0,00008 \\
0,0006\end{array}$ & $\begin{array}{l}0,0000005 \\
0,000003\end{array}$ & $\begin{array}{l}4 \\
0,08\end{array}$ & $\begin{array}{l}74 \\
65\end{array}$ \\
\hline Fin d'égouttage & 3 & 0,99 & 1,00 & 0,008 & 0,0001 & 0,005 & 0,30 \\
\hline Salle froide & 4 & 0,98 & 1,00 & 0,01 & 0,00006 & 0,00007 & 2 \\
\hline 3 jours après salage & 11 & 0,99 & 1,00 & 0,003 & 0,002 & 0,06 & 0,01 \\
\hline 10 jours après salage & 23 & 0,62 & 1,00 & 0,11 & 0,002 & 0,000001 & 0,02 \\
\hline Premier affinage & 28 & 0,29 & 1,00 & 0,34 & 0,006 & 0,000004 & 0,01 \\
\hline Plombage & 35 & 0,23 & 1,00 & 0,22 & 0,01 & 0,0005 & 0,01 \\
\hline 15 jours après plombage & $\begin{array}{r}49 \\
65 \\
94 \\
142\end{array}$ & $\begin{array}{l}0,30 \\
0,15 \\
0,20 \\
0,50\end{array}$ & $\begin{array}{l}0,50 \\
1,00 \\
1,00\end{array}$ & $\begin{array}{l}0,69 \\
0,30 \\
0,37 \\
0,22\end{array}$ & $\begin{array}{l}0,44 \\
0,02 \\
0,0003 \\
0,002\end{array}$ & $\begin{array}{c}0,2 \\
2,3 \\
12 \\
2,0\end{array}$ & $\begin{array}{c}0,04 \\
87 \\
0,55 \\
0,29\end{array}$ \\
\hline Vente & 197 & 0,75 & 1,00 & 0,31 & 0,0003 & 2,8 & 0,38 \\
\hline
\end{tabular}


Pendant les premières $48 \mathrm{~h}$, qui correspondent à l'acidification et à l'égouttage, les bactéries acidifiantes représentent pratiquement la totalité de la flore. Les microcoques sont plus nombreux que les levures. Mais tous les types de levures et de bactéries sont en multiplication active. A $48 \mathrm{~h}$, le $\mathrm{pH}$ a atteint pratiquement son point le plus bas et l'acidification peut être considérée comme terminée. Les levures deviennent plus nombreuses que les microcoques en surface. Cette inversion de la flore ne se produit pas au centre où l'anaérobiose est nettement plus prononcée ; elle n'interviendra que beaucoup plus tard en fin d'égouttage. Les souches assimilant le nitrate ont présenté une multiplication beaucoup plus rapide que les autres levures, sur les faces comme au centre du fromage. Pendant cette période, les levures acidifiantes représentent une fraction notable de la flore de levure mais les populations semblent varier d'un fromage à un autre. Ces diverses tendances de l'évolution de la flore continuent jusqu'au salage. Il faut noter également que la proportion des levures sporulées augmente pendant la phase d'acidification et d'égouttage ; cette tendance est retardée au centre par rapport aux faces. Rappelons qu'à ces divers stades, les souches sporulées appartiennent surtout au genre Hansenula (nitrate +), plus rarement au genre Saccharomyces (nitrate-), exceptionnellement au genre Debaryomyces (nitrate-). De nombreuses souches assimilant le nitrate et très proches des Hansenula ont été aussi trouvées mais elles ne fermentent pas les sucres. Les souches anascosporées appartiennent en général au genre Torulopsis, mais souvent aussi aux genres Candida et Rhodotorula.

Diverses souches appartenant à d'autres genres ont été trouvées et sont à l'étude; elles ne sont pas signalées ici car elles sont trop rares dans le fromage pour présenter un intérêt industriel.

Le salage a des conséquences surtout en surface. Pratiquement, tous les types de levures et de bactéries diminuent de nombre. Les levures sporulées deviennent de plus en plus rares après le salage. De même, les souches qui n'assimilent pas le nitrate et les souches à pouvoir fermentaire sont éliminées. On observe aussi une baisse du pourcentage de souches productrices d'acides. La flore dominante est constituée pendant le premier affinage par des Hansenula et par des Torulopsis qui réduisent aussi le nitrate. Les souches les plus nombreuses sont des Torulopsis nitrate + à métabolisme exclusivement oxydatif. Les souches des autres genres représentent moins de 1 p. 100 de la flore de la cuve. Pendant cette période, les bactéries acidifiantes perdent rapidement de leur importance et il en est de même des microcoques. Les levures représentent jusqu'à 34 p. 100 de la flore totale. Il est évident que l'importance technologique d'une souche n'est pas nécessairement une fonction exclusive de sa fréquence. Cependant, il est nécessaire d'insister sur le fait que les Torulopsis assimilant le nitrate et à métabolisme exclusivement oxydatif constituent la flore dominante au moment même où les levures deviennent le constituant essentiel de la flore en surface. 
Il est probable qu'ils constituent aussi un élément important du révérun.

Au centre, le salage a des conséquences plus atténuées. Le rapport levure/flore totale augmente et passe de 0,002 à 0,01 . Les levures ne sont donc pas aussi nombreuses qu'en surface mais peuvent jouer un rôle actif notamment dans l'oxydation de l'acide lactique. Le piquage qui suit le salage a provoqué une augmentation du taux d'oxygène à l'intérieur du fromage et a certainement favorisé le développement des levures. Les bactéries acidifiantes restent dominantes et constituent près de 100 p. 100 de la flore totale. Les levures sporulées deviennent moins nombreuses : les Saccharomyces disparaissent pratiquement et seules les Hansenula subsistent. Parmi les anascosporées, les Torulopsis incapables d'assimiler le nitrate, les Candida, les Rhodotorula ont tendance à disparaître. Seules subsistent, en quantités importantes, les Torulopsis assimilant les nitrates ; parmi ces dernières celles qui ont un métabolisme exclusivement oxydatif deviennent de plus en plus nombreuses mais restent minoritaires. En définitive, le type dominant correspond aux Torulopsis à pouvoir fermentaire et assimilant le nitrate. La flore de levure est plus variée au centre qu'en surface et le type dominant en surface n'arrive ici qu'en troisième position.

Le plombage correspond à la mise du fromage sous une pellicule d'étain ; cette opération a pour but de ralentir les échanges gazeux et d'augmenter l'anaérobiose.

En surface, les bactéries acidifiantes continuent à perdre de leur importance. Les levures constituent environ un tiers de la flore totale. Un fait important : à partir du plombage le rapport microcoques/levures devient $>1$. La flore totale et la flore de levure diminuent alors que les microcoques entrent dans une phase de multiplication très active. L'anaérobiose est probablement responsable de ces phénomènes. Parmi les levures, on assiste à la disparition des types sporulés, des souches qui n'assimilent pas le nitrate et des souches à métabolisme fermentaire. Les souches à pouvoir acidifiant disparaissent également. Pratiquement, toutes les souches isolées après plombage sont des Torulopsis à métabolisme exclusivement oxydatif qui assimilent le nitrate. II est vraiment curieux de constater que, dans un milieu pauvre en oxygène, les levures incapables de fermenter les glucides constituent la totalité de la flore de levure et le tiers de la-flore totale. Le métabolisme de ces souches devra être étudié en détail, d'autant plus que leur importance technologique est indiscutable.

Au centre, après plombage, les bactéries acidifiantes constituent toujours la flore dominante. Les levures sont peu nombreuses et leur nombre diminue comme d'ailleurs diminue la flore totale. Cependant, les levures restent plus nombreuses que les microcoques. En effet, après une multiplication très rapide, les microcoques diminuent. Il se peut d'ailleurs que ces variations soient dues à 
l'hétérogénéité entre les pains d'un même cuvier. La flore reste plus variée qu'en surface. Les trois types dominants sont constitués par les Hansenula, les Torulopsis assimilant les nitrates et fermentant les glucides et les Torulopsis assimilant les nitrates à métabolisme exclusivement oxydatif. Cette flore est très voisine de celle observée avant plombage.

En définitive, la flore de levure est très variée jusqu'au salage, c'est-à-dire pendant la première semaine de fabrication. Sous l'effet des diverses opérations technologiques (salage et piquage) une flore dominante s'établit pour toute la durée de l'affinage : Torulopsis nitrate + fermentation - en surface, et un mélange d'Hansenula et de Torulopsis nitrate + au centre du fromage. La différence fondamentale entre face et centre est l'abondance de types à pouvoir fermentaire au centre du fromage. Le plombage ne fait qu'accentuer la sélection commencée au salage.

L'étude technologique et biochimique de certaines souches devra être entreprise. Les levures productrices d'acides doivent jouer un rôle important dans le développement de la flaveur du Roquefort. Il est probable que deux types de métabolismes très différents seront trouvés selon que l'on considérera les souches à pouvoir fermentaire ou les souches à métabolisme exclusivement oxydatif. Il faudra également étudier en détail le métabolisme des Hansenula et des Torulopsis du Roquefort pour déterminer exactement leur rôle dans l'évolution et l'affinage du fromage.

\section{Rés u m é}

2484 souches de levures ont été isolées pendant la fabrication et l'affinage d'un cuvier de Roquefort. Nous avons fait, parallèlement, sur les faces et le centre des fromages, des analyses bactériologiques (flore totale, coliformes, microcoques, staphylocoques) et des analyses chimiques $\left(\mathrm{pH}\right.$, extrait sec, $\mathrm{O}_{2}, \mathrm{CO}_{2}$, lactose, acide lactique). Les résultats ont montré que l'on pouvait définir dans l'évolution d'un fromage de Roquefort, un nombre précis de phases, chacune séparée de la suivante par une opération technologique : acidification, salage, piquage, pliage du fromage sous étain. Les levures isolées sur 8 milieux de composition différente (2 sources de carbone, 2 pH, 2 taux de sel) ont été classées en types physiologiques.

Dans les premières phases de la fabrication (acidification et égouttage) les levures sporulées sont plus abondantes que les levures non sporulées. Dès la fin de la phase d'acidification, les levures du type Hansenula et à un degré moindre du type Saccharomyces, se multiplient activement sur les faces et deviennent plus nombreuses que les microcoques. Au centre, cette évolution est retardée car les teneurs en gaz carbonique atteignent 80 p. 100. Parmi les anascosporées, le genre Torulopsis est le plus représenté. 
Le salage provoque, sur les faces, la disparition de 90 p. 100 de la flore de levure. Les types sporulés deviennent plus rares, les souches qui n'assimilent pas le nitrate et qui fermentent le glucose disparaissent. A partir du salage, les Torulopsis assimilant le nitrate et à métabolisme strictement oxydatif constituent la flore dominante, et cela au moment où les levures deviennent le constituant essentiel de la flore de surface (formation du "révérun " ou morge).

Au centre, l'action du sel est retardée. Ce qui est important c'est le piquage qui suit le salage : il y a augmentation du taux d'oxygène au centre, et les levures se multiplient activement en déshydrogénant l'acide lactique (le rapport levure/flore totale passe de 0,002 à 0,01 ). Les levures sporulées diminuent, seules subsistent les Hansenula. Le type dominant correspond aux Torulopsis à pouvoir fermentaire et assimilant le nitrate.

A partir du "plombage» (pliage sous étain), il y a inversion de flore en surface, les microcoques deviennent plus nombreux que les levures. Le seul type physiologique qui pratiquement subsiste correspond aux Torulopsis à métabolisme oxydatif et qui assimilent le nitrate (type Torulopsis aeria). Au centre, le nombre des levures diminue, mais elles restent plus nombreuses que les microcoques : on retrouve trois types dominants : Hansenula, Torulopsis assimilant le nitrate, d'une part à métabolisme fermentatif, d'autre part à métabolisme oxydatif. Le premier jour de fabrication nous avons déterminé 18 types physiologiques de levures sporulées et 24 types de levures non sporulées; à la fin de l'affinage on ne retrouve que 2 types physiologiques de sporulées et 3 types de non sporulées au centre, et 3 types de non sporulées sur les faces.

\section{S u m m a ry}

2484 strains of yeasts were isolated during the production and ripening of a vat of Roquefort cheese. Both bacteriological analyses of the surface and centre of the cheeses (total flora, coliform, micrococci, staphylococci) and chemical analyses $\left(\mathrm{pH}\right.$, dry matter, $\mathrm{O}_{2}, \mathrm{CO}_{2}$, lactose, lactic acid) were carried out. The résults indicated that it was possible to follow the development in the cheese of a number of distinct stages, each apart from the other, by means of technological process : acidification, salting, piercing, wrapping cheese in tin-foil. The yeasts were isolated on 8 media of differing classes ( 2 different carbone bases, $2 \mathrm{pH}$ for each, 2 salt content), and were classified according to their physiological caracteristics.

During the first stage of the production (acidification and whey draining), the spore-forming yeasts were more abundant than the non spore-forming ones. Immediately following the acidification stage, the yeasts of the genus Hansenula and to a less extent the genus Saccharomyces, actively multiplied on the surface and were more numerous than the micrococci. In the centre, this development 
was retarded because the carbon dioxide content had attained a level of 80 per cent. Among the non spore-forming species, the genus Torulopsis was the most apparent.

The salting caused the disappearance of 90 per cent of the yeast flora on the surface. The spore-forming species were fewer and the strains which were unable to assimilate nitrate and ferment glucose disappeared. After salting, the Torulopsis species, which assimilated nitrate in a strictly oxidative manner, were the dominating flora and at a time when the yeasts had become the main constituent of the surface flore (formation of slime).

In the centre, the salt action was retarded. The important factor being the piercing which follows the salting : increase of oxygen content in the centre and active multiplication of the yeasts with the dehydrogenation of the lactic acid (the ratio yeasts/total flora changed from 0,002 to 0,01$)$. The spore-forming yeasts decreased and only the Hansenula species remained. The dominating species belonging to the fermentative and nitrate assimilating species of Torulopsis.

After wrapping in tin-foil, there was an inversion of surface flora with more numerous micrococci than yeast. The only physiological species which practically remained belonged to the oxidative and nitrate assimilating species of Torulopsis (Torulopsis aeria). In the centre, the number of yeasts decreased but remained more numerous than the micrococci : three dominant species were found: Hansenula, nitrate assimilating Torulopsis of fermentative nature on one hand and oxidative on the other hand. On the Ist. day of production, we estimated 18 physiological species of spore-forming yeasts and 24 non spore-forming ; at the end of ripening, only 2 physiological species of spore-forming yeasts were observed, 3 non spore-forming in the centre and 3 non spore-forming on the surface.

\section{Bibliographie}

[1] Bret (G.) et Congras (A.) (1963-1964) 1 er essai du 15-5-1963. - Résultats du $1^{\text {er }}$ essai (note du 16-10-1963). Résultats du $2^{\text {me }}$ essai (note du 20-1-1964). Travaux et notes non publiés.

[2] Campbell (I.) (1968), - Identification of yeasts. Process Biochemistry, May.

[3] Devoyod (J. J.) (1969), - La flore microbienne du fromage de Roquefort. II. Staphylocoques et microcoques. Le Lait, 481-482, p. 20.

[4] Devoyod (J. J.) et Muller (M.) (1969). - La flore microbienne du fromage de Roquefort. III. Les Streptocoques lactiques et les Leuconostocs. Le Lait, 487, p. 369. Communication personnelle.

[5] Devoyod (J. J.) et Muller (M.) (1969). - La flore microbienne du fromage de Roquefort. IV. Les Entérocoques. Le Lait, 489-490, p. 637. Communication personnelle.

[6] Devoyod (J. J.), BRET (G.) (1966). - Evolution de la flore microbienne au cours de la fabrication et de l'affinage du fromage de Roquefort. $X V I I^{m e}$ Cong. Int. de Laiterie D-2, p. 585. 
[7] Devoyod (J. J.), Bret (G.) et Auclair (J. E.) (1968). Flore microbienne du fromage de Roquefort. I. Son évolution au cours de la fabrication et de l'affinage. Le Lait, 479-480, p. 613.

[8] Dolezalek (J.), Bartuskova (M.) (1966). - A study on the microflora and ripining of "Blue cheese ". XVII ${ }^{m e}$ Cong. Int. de Laiterie D-1, p. 151.

[9] Fowell (R.) (1967). - Factors controlling the sporulation of yeasts. II. The sporulation phase. J. Appl. Bact., 30 (3), p. 450.

[10] Harper (W. J.), Randolph (H. E.) (1960). - Lactic acide in Cottage cheese. American Milk Review, 22 (6), p. 43-46.

[11] Hoffman (W. S.). - A rapid photoelectric method for the determination of glucose in blood and urine. J. Biol. Chem., $120: 51,1937$.

[12] Knudsen (S.), Overby (A. J.) (1942). - Kong. Vet. Og. Land. Darskz, Copenhague.

[13] Lenoir (J.), Auberger (B.) (1966). - Contribution à l'étude de la flore microbienne du fromage de type Camembert. XVII me Cong. Int. de Laiterie D-2, p. 595.

[14] LENOIR (J.) (1962). - La flore microbienne du camembert et son évolution au cours de la maturation. C.R. Ac. Agr., 48, p. 392.

[15] Ling (E. R.) (1951). - Détermination de l'acide lactique dans le lait. J. Sc. Agr., 2, p. 279.

[16] Lodder (J.) et Kreger-VAN Riz (N. J. W.) (1952). - The Yeasts, a Taxonomic Study. Amsterdam, N. Holland Publ. Co.

[17] MAXA (V.), JiCINCKY (V.) (1956). - A study of some asporogenous yeasts in view of their application in the manufacture of Roquefort cheese. $X I V^{\text {me }}$ Cong. Int. de Laiterie S-2, p. 345.

[18] Mocouot (G.) et Bejambes (M.) (1960). - Technology of ewe's and goat's milk products. Dairy Sc. Abstr., 22, p. 1-12.

[19] Proks (J.), Dolezalek (J.) et Pech (Z.) (1959). - A study of the coaction of the yeasts of the genus Torulopsis on the formation of the methyl ketones in the cheese of Roquefort type. $X V^{m e}$ Cong. Int. de Laiterie, 2, p. 729.

[20] Sode-Mogensen (M. T.) (1947). - Medel. Statens Mejeriforsok, p. 21.

[21] Somogyi (M.) (1952). - J. Biol. Chem., 195, p. 19-23.

[22] Wilster (G. M.), Price (W. V.), Murris (A. J.), Goss (E. F.) and Sanders (1937). - J. Dairy Sc., p. 20-27.

Reçu pour publication en août 1969. 